\title{
Méthode analytique de conduction inverse appliquée à la mesure du coefficient de transfert local sur un cylindre en convection forcée
}

\author{
D. Maillet et A. Degiovanni \\ Laboratoire LEMTA-CNRS U.A. 875, Ecole des Mines, Parc de Saurupt, 54000 Nancy, France
}

(Reçu le 12 décembre 1988, accepté le 29 mars 1989)

\begin{abstract}
Résumé. - Les méthodes directes de mesure du coefficient local de transfert de chaleur sur une paroi présentent des limitations intrinsèques. Une méthode inverse, basée sur un modèle analytique de conduction de la chaleur dans un tube cylindrique, est développée. Elle permet, à partir des seules mesures de température à l'intérieur du cylindre, de remonter au profil de coefficient de transfert en surface. Des résultats expérimentaux, cohérents avec ceux de la littérature, sont présentés.
\end{abstract}

Abstract. - Direct methods of surface heat-transfer coefficient measurement present specific limitations. An inverse method, based on an analytical heat-conduction model for a cylindrical pipe is developped. Calculation of the surface heat-transfer coefficient profile only requires temperature measurements inside the cylinder. Experiments have been made. The results which are presented seem to be coherent with those of other authors.

\section{Introduction.}

La mesure du coefficient local de transfert de chaleur $h$ par une méthode directe (ni optique, ni analogique) entre un point $M$ d'une paroi et un fluide à la température $T_{\mathrm{e}}$, pose des problèmes de métrologie car il faut mesurer au même endroit une température de surface $T$ et un flux de chaleur $\phi$ qui traverse une portion $\Delta S$ de la surface centrée sur M (Fig. 1).

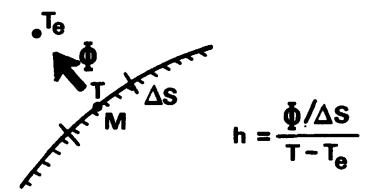

Fig. 1. - Mesure directe du coefficient de transfert.

[Direct measurement of heat-transfer coefficient.]

Dans le cas d'une géométrie cylindrique, typique par exemple d'un échangeur de chaleur à courants croisés, le problème est facilité car la sonde de température peut être déplacée angulairement par une rotation du cylindre.
Le flux est généralement créé par une résistance électrique qui est, soit pelliculaire entourant le cylindre (flux uniforme) [1], soit localisée en $M$ avec des gardes et compensations et une génération de chaleur sur l'axe [2]. Dans ces expériences la sonde de température ne peut mesurer avec précision la température de surface si celle-ci n'est pas uniforme. En outre, l'évaluation des pertes thermiques est délicate.

Une autre méthode n'a, à notre connaissance, pas été étudiée ; elle consiste, à partir d'une température mesurée au cœur du cylindre et d'une condition limite interne imposée et connue, à remonter à la condition limite de Fourier sur la surface externe du cylindre, c'est-à-dire au coefficient $h$.

\section{Modèle analytique du problème direct.}

2.1 Modélisation. - Dans le problème direct, on cherche à calculer la température $T$ en un point $\mathrm{P}$ d'un tube cylindrique de coordonnées polaires $(r, \phi)$ compris entre 2 surfaces cylindriques de rayons $r_{1}$ et $r_{2}$ (Fig. 2). On impose une température uniforme sur le rayon intérieur $r_{1}$ et un coefficient de transfert $h$ variant avec l'abscisse angulaire $\phi$ sur la surface extérieure $r_{2}$. On s'impose en outre une 
symétrie de $h$ par rapport au plan $\phi=0$. La température est alors solution de l'équation de la chaleur et des conditions limites suivantes :

$$
\frac{\partial^{2} T}{\partial r^{2}}+\frac{1}{r} \frac{\partial T}{\partial r}+\frac{1}{r^{2}} \frac{\partial^{2} T}{\partial \phi^{2}}=0
$$

$$
\begin{cases}T \text { fonction paire en } \phi & \\ T \text { périodique de période } 2 & \pi \text { en } \phi \\ T=T_{1} & \text { en } \quad r=r_{1} \\ -\lambda \frac{\partial T}{\partial r}=h(\phi)\left[T-T_{\mathrm{e}}\right] & \text { en } \quad r=r_{2} .\end{cases}
$$

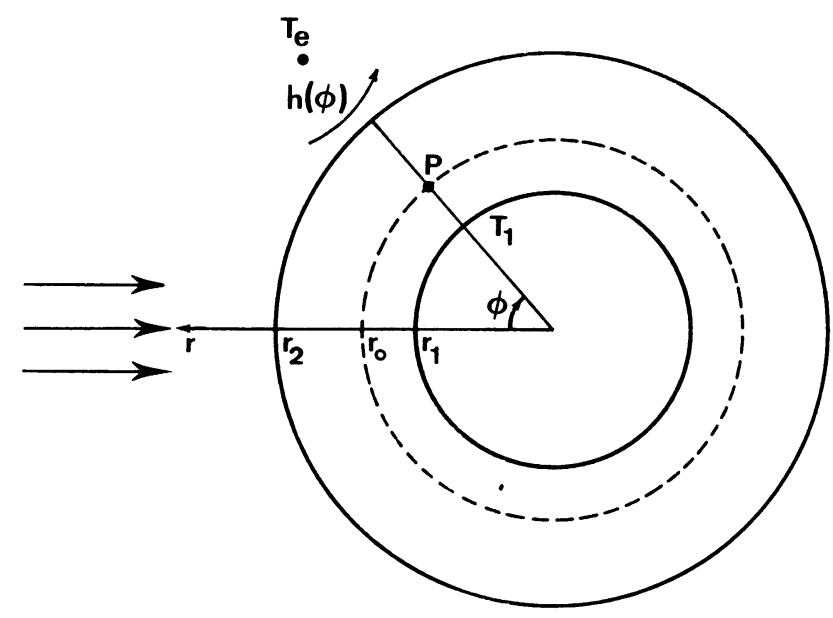

Fig. 2. - Modélisation.

[Modelling of the problem.]

La dernière condition (5) interdit l'utilisation de la méthode de séparation des variables à cause de la non constance de $h$. Pour pallier à cet inconvénient, il est possible d'utiliser la méthode fluxmétrique [3] qui se rapproche de la méthode « Heat Flux Based Unsteady Surface Element » développée par Keltner et Beck [4] en régime transitoire. Elle consiste, dans un premier temps, à imposer à la place de (5) une condition de flux variable sur la surface extérieure :

$$
-\lambda \frac{\partial T}{\partial r}=\varphi_{2}(\phi) \text {. }
$$

Le problème peut alors être résolu en exprimant la temperature $T$ en fonction de $\varphi_{2}(\phi)$. Cette densité de flux sera identifiée dans un deuxième temps en égalant les membres de droite des conditions (5) et (6).

Pour rendre homogène la condition (4), on fait un changement de fonction :

$$
\theta(r, \phi)=T_{1}-T(r, \phi) .
$$

Le problème est alors linéaire, à coefficients constants et homogène, à l'exception de la condition (6). Il est résolu par séparation des variables [5]:

$$
\theta(r, \phi)=\mu \sum_{n=0}^{\infty} a_{n} g_{n}(x) \cos n \phi
$$

avec

$$
\begin{aligned}
\mu & =2 r_{2} / \lambda \pi \\
x & =r / r_{2} \\
a_{n} & =\int_{0}^{\pi} \varphi_{2}\left(\phi^{\prime}\right) \cos n \phi^{\prime} \mathrm{d} \phi^{\prime} \\
g_{0}(x) & =\frac{1}{2} \ln x / k \\
g_{n}(x) & =\frac{1}{n x^{n}} \frac{x^{2 n}-k^{2 n}}{1+k^{2 n}} \quad \text { si } n>1 \\
k & =r_{1} / r_{2} .
\end{aligned}
$$

2.2 RÉSOlUtion EXACTE. $-\varphi_{2}$ est alors solution de l'équation intégrale suivante :

$$
\varphi_{2}(\phi)=h(\phi)\left[\theta_{\mathrm{e}}-\theta_{2}(\phi)\right]
$$

avec :

$$
\begin{aligned}
\theta_{\mathrm{e}} & =T_{1}-T_{\mathrm{e}} \\
\theta_{2}(\phi) & =\theta\left(r_{2}, \phi\right)
\end{aligned}
$$

ou encore, en tenant compte de l'équation (8):

$$
\varphi_{2}(\phi)-\mu \int_{0}^{\pi} Q\left(\phi, \phi^{\prime}\right) \varphi_{2}\left(\phi^{\prime}\right) \mathrm{d} \phi^{\prime}=f(\phi)
$$

avec :

$$
\begin{aligned}
Q\left(\phi, \phi^{\prime}\right) & =\sum_{n=0}^{\infty} \alpha_{n}(\phi) \beta_{n}\left(\phi^{\prime}\right) \\
\alpha_{n}(\phi) & =-g_{n}(1) h(\phi) \cos n \phi \\
\beta_{n}\left(\phi^{\prime}\right) & =\cos n \phi^{\prime} \\
f(\phi) & =\theta_{\mathrm{e}} h(\phi) .
\end{aligned}
$$

L'équation (11) est une équation intégrale à noyau dégénéré [6] dont la solution est la suivante :

$$
\varphi_{2}(\phi)=\mu \sum_{n=0}^{\infty} z_{n} \alpha_{n}(\phi)+f(\phi)
$$

les coefficients $z_{n}$ étant solution du système suivant :

$$
z_{n}-\mu \sum_{l=0}^{\infty} t_{n l} z_{l}=f_{n} \quad(n=0, \infty)
$$

avec :

$$
\begin{aligned}
& t_{n l}=\int_{0}^{\pi} \beta_{n}\left(\phi^{\prime}\right) \alpha_{l}\left(\phi^{\prime}\right) \mathrm{d} \phi^{\prime} \\
& f_{n}=\int_{0}^{\pi} \beta_{n}\left(\phi^{\prime}\right) f\left(\phi^{\prime}\right) \mathrm{d} \phi^{\prime} .
\end{aligned}
$$

La solution analytique exacte $\theta(r, \phi)$ est alors obtenue en remplaçant $\varphi_{2}(\phi)$, par son expression (12) dépendant de $h(\phi)$ dans la définition (9) des coefficients $a_{n}$ de la série de Fourier (8). 
2.3 RÉSOlUTION APPROCHÉE. - Dans la pratique, le calcul des coefficients $z_{n}$ entrant dans l'écriture (12) de $\varphi_{2}$ nécessite la résolution du système linéaire (13) comportant un grand nombre d'équations (idéalement l'infini), ce qui peut poser un problème de conditionnement de matrice lorsqu'on augmente la borne supérieure de $n$. Par ailleurs, lorsque l'on va examiner le cas du problème inverse, les valeurs recherchées pour $\varphi_{2}$ et $h$ sur $[0, \pi]$ ne pourront plus être continues. Nous nous proposons d'examiner le cas particulier où la fonction $\varphi_{2}$ est constante par morceaux :

$$
\begin{array}{rll}
\varphi_{2}(\phi)=\varphi_{2 i} & \text { pour } & \phi_{i-1} \leqslant \phi<\phi_{i} \\
& \text { avec } & \phi_{i}=i \frac{\pi}{N} \\
& \text { et } & 1 \leqslant i \leqslant N .
\end{array}
$$

Les coefficients $a_{n}$ peuvent alors être exprimés en fonction des valeurs discrètes $\varphi_{2 i}$ :

avec

$$
\begin{aligned}
& a_{0}=\frac{\pi}{N} \sum_{i=1}^{N} \varphi_{2 i} \\
& a_{n}=\sum_{i=1}^{N} \frac{\Delta_{n i}}{n} \varphi_{2 i} \text { si } n>0
\end{aligned}
$$

La température $\theta$ est alors une combinaison linéaire des densités de flux $\varphi_{2 i}$ :

$$
\theta(r, \phi)=\sum_{i=1}^{N} d_{i} \varphi_{2 i}=[\tilde{d}]\left[\varphi_{2}\right]
$$

avec :

$d_{i}(x, \phi)=\mu\left[\frac{\pi}{N} g_{0}(x)+\sum_{n=1}^{\infty} \frac{1}{n} \Delta_{n i} g_{n}(x) \cos n \phi\right]$.

On définit le coefficient de transfert moyen $h_{i}$ sur l'intervalle $\left[\phi_{i-1}, \phi_{1}\right]$ comme le rapport de la densité moyenne de flux à l'écart moyen de température à la paroi :

$$
h_{i}=\varphi_{2 i} /\left(\theta_{\mathrm{e}}-\bar{\theta}_{2 i}\right)
$$

avec :

$$
\bar{\theta}_{2 i}=\frac{N}{\pi} \int_{\phi_{i-1}}^{\phi_{i}} \theta\left(r_{2}, \phi\right) \mathrm{d} \phi
$$

$\bar{\theta}_{2 i}$ peut être calculé par intégration de (14) en $x=1$ :

$$
\bar{\theta}_{2 i}=\sum_{i=1}^{N} C_{i j} \varphi_{2 j}
$$

avec :

$$
C_{i j}=\mu\left[\frac{\pi}{N} g_{0}(1)+\frac{N}{\pi} \sum_{n=1}^{\infty} \frac{1}{n^{2}} g_{n}(1) \Delta_{n i} \Delta_{n j}\right] .
$$

En combinant les relations (15) et (16), les densités de flux sont solution de l'équation :

$$
A\left[\varphi_{2}\right]=[u]
$$

avec :

$$
\begin{aligned}
A & =(C+H) / \theta_{\mathrm{e}} \\
H_{i j} & =\delta_{i j} / h_{i} \quad \text { pour } 1 \leqslant i \leqslant N \\
u_{i} & =1 \\
\delta_{i j} & \text { étant le symbole de Kronecker } .
\end{aligned}
$$

La solution approchée du problème direct est donc :

$$
\theta(r, \phi)=[\tilde{d}] A^{-1}[u]
$$

2.4 Résultats DU MODÈle DiRECT. - Pour valider le modèle direct, examiner la faisabilité de la méthode inverse et étudier l'influence du pas de discrétisation angulaire, nous nous sommes donnés une fonction $h(\phi)$ représentative du refroidissement d'un cylindre, de $32 \mathrm{~mm}$ de diamètre extérieur, par un écoulement forcé d'air en régime subcritique :

- $h$ constant égal à $250 \mathrm{~W} \mathrm{~m}^{-2} \mathrm{~K}^{-1}$ sur $\left[0,40^{\circ}\right]$

- décroissance linéaire de $h$ jusqu'à $100 \mathrm{~W}$ $\mathrm{m}^{-2} \mathrm{~K}^{-1}$ sur $\left[40^{\circ}, 80^{\circ}\right]$

- croissance linéaire de $h$ jusqu'à $200 \mathrm{~W}$ $\mathrm{m}^{-2} \mathrm{~K}^{-1}$ sur $\left[80^{\circ}, 180^{\circ}\right]$.

Le rayon intérieur est de $8 \mathrm{~mm}$ - rapport de forme $k$ de $0,5-$ la température est calculée pour un rayon $r_{0}$ de $14 \mathrm{~mm}$ et la conductivité thermique du matériau constituant le tube est de $0,26 \mathrm{~W} \mathrm{~m}^{-1} \mathrm{~K}^{-1}$. L'écart de température extrême $\theta_{\mathrm{e}}$ du système est de $40^{\circ} \mathrm{C}$.

Les résultats du modèle sont représentés (Figs. 3a, $b$ et c) pour des discrétisations sur 5,9 et 18 intervalles. Les $N$ coefficients $h_{i}$ (entrée du modèle) sont les moyennes sur chaque intervalle de la fonction $h(\phi)$ donnée plus haut.

Il est à noter que les variations de température sont mesurables - de l'ordre de $2,5^{\circ} \mathrm{C}$ - et que la sensibilité du profil de $\theta_{0}$ au nombre d'intervalles $N$ est faible. Lorsque l'on donne à $N$ les valeurs successives suivantes : $5,9,18$ et 36 , l'écart maximum local passe de 15 à 12 puis 2 centièmes de degré Celsius tandis que l'écart quadratique moyen passe de 7,2 à 3 puis 0,5 centièmes de degré Celsius, ce qui nous a permis de travailler avec une valeur optimum de 18 intervalles.

\section{Modèle inverse.}

3.1 CAS DE MESURES EXACTES. - Dans ce problème, les conditions aux limites (2), (3) et (4) sont inchangées et l'on remplace la condition (5) par une condition de température imposée en un nombre 


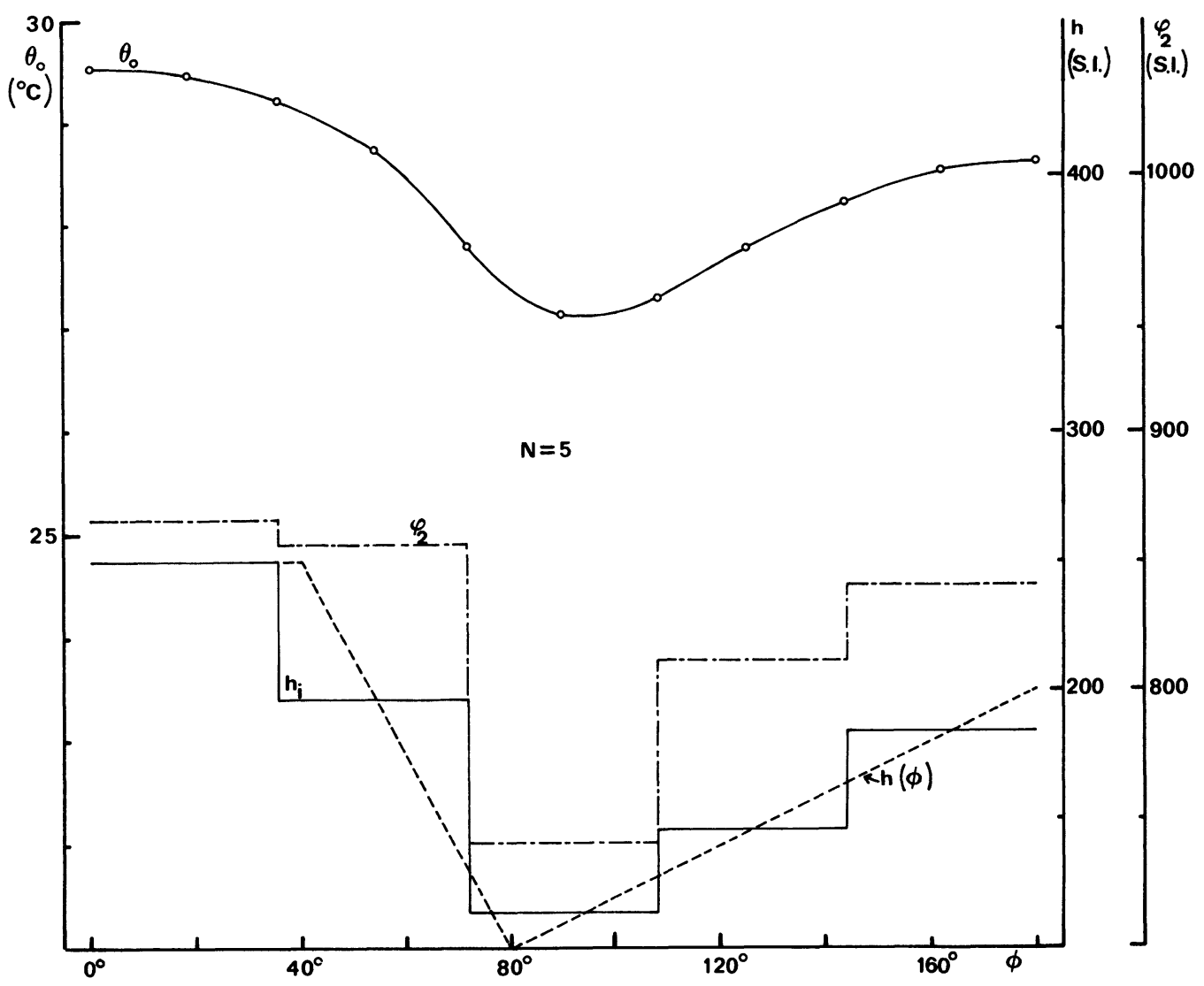

a)

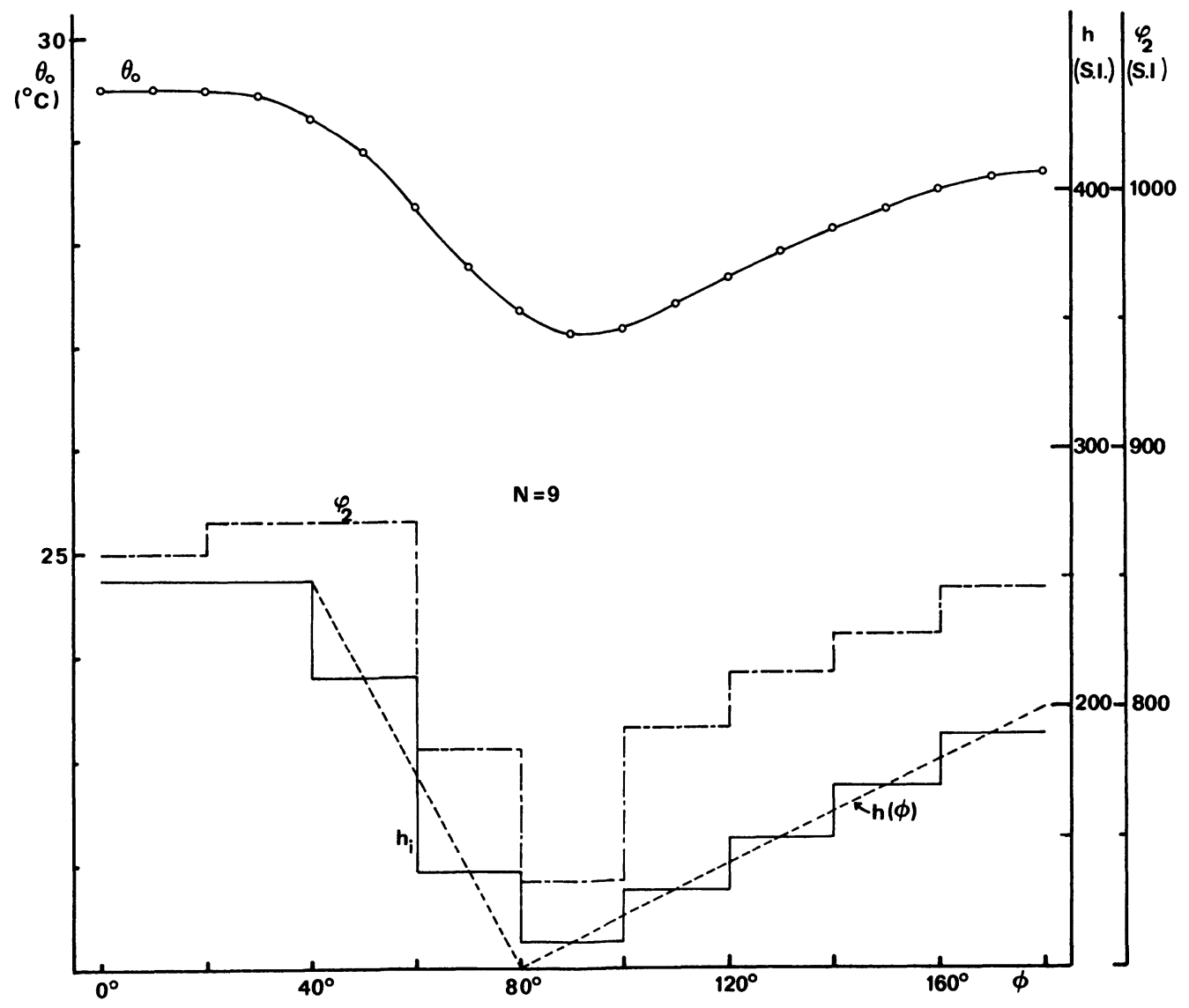

b) 


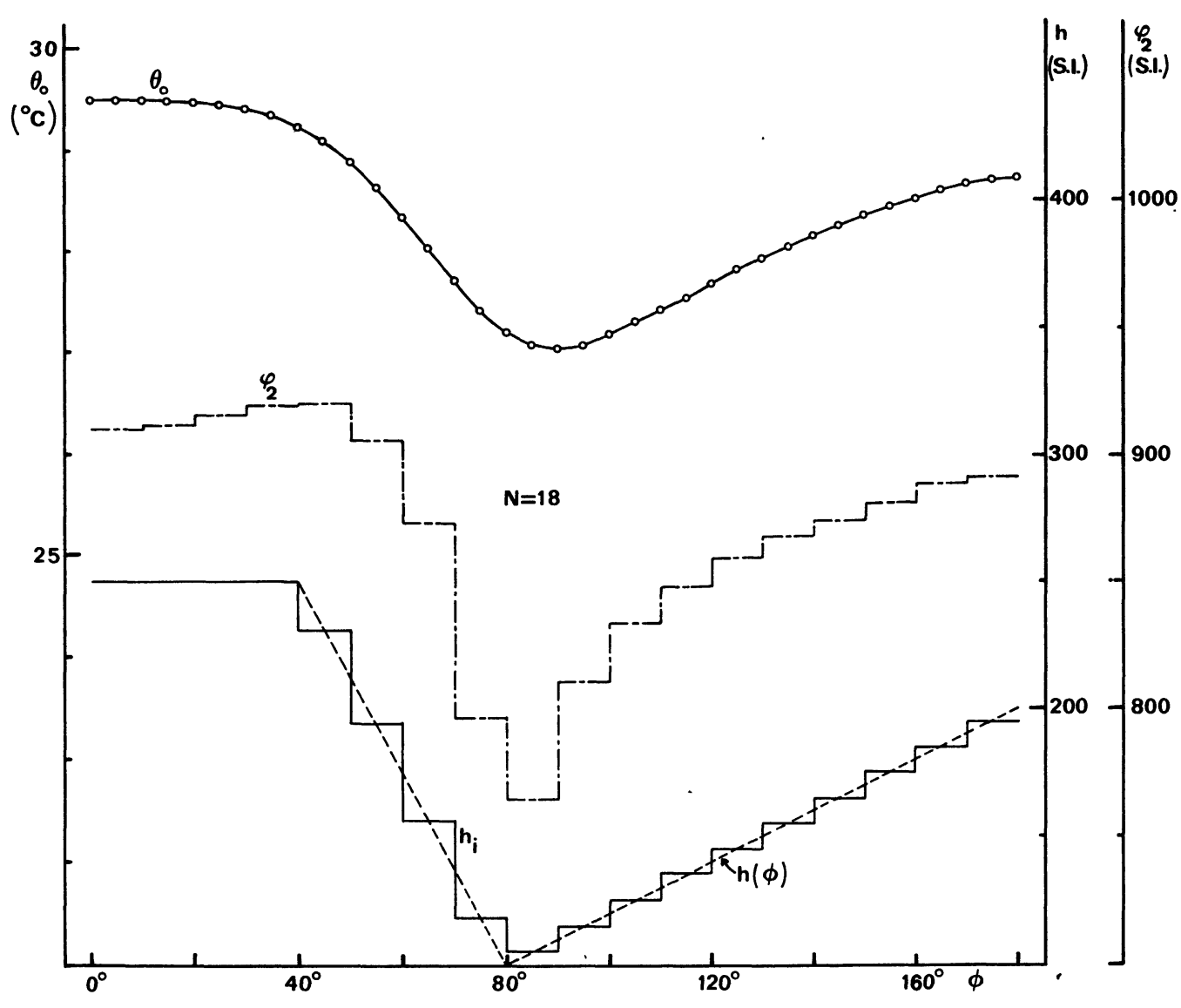

c)

Fig. 3. - Résultats du modèle direct, influence du pas de discrétisation angulaire, a) 5 intervalles ; b) 9 intervalles ; c) 18 intervalles.

[Direct model results, influence of the angular discretization step, a) 5 intervals ; b) 9 intervals ; c) 18 intervals.]

$(N+1)$ de points sur un rayon $r_{0}$ du tube :

$$
\theta\left(r_{0}, \phi_{i}\right)=\theta_{0 i} \text { pour } 0 \leqslant i \leqslant N
$$

avec :

$$
r_{1}<r_{0}<r_{2} \text {. }
$$

La relation (14) s'écrit alors :

$$
\theta_{0 i}=\sum_{j=1}^{N} d_{j}\left(x_{0}, \phi_{i}\right) \varphi_{2 j}
$$

avec :

$$
x_{0}=r_{0} / r_{2}
$$

ou encore :

$$
\left[\theta_{0}\right]=B\left[\varphi_{2}\right]
$$

avec :

$$
B_{i j}=d_{j}\left(x_{0}, \phi_{i}\right)
$$

Si les mesures des $\theta_{0 i}$ sont exactes, il suffit de résoudre le système (21) pour calculer les densités de flux $\varphi_{2 j}$. Il est ensuite possible de calculer les $N$ valeurs $h_{i}$ du coefficient de transfert à l'aide de (15) et (16).

3.2 CAS de Mesures bruttées. - Dans la réalité, les mesures expérimentales sont toujours bruitées et dans ce cas la méthode d'inversion présentée en 3.1 n'est plus valable. Nous appelons $y_{i}$ la valeur de $\theta$ mesurée au rayon $r_{0}$ et à l'angle $\phi_{i}$ et $\theta_{0 i}$ la valeur exacte correspondante, $\varepsilon_{i}$ étant l'erreur aléatoire supposée additive :

$$
y_{i}=\theta_{0 i}+\varepsilon_{i} .
$$

Pour prendre en compte ce bruit il est possible d'utiliser une méthode des moindres carrés qui consiste à minimiser la somme $S$ des écarts quadratiques $\varepsilon_{i}^{2}$ par rapport aux flux $\varphi_{2 i}$ recherchés. L'inconvénient de cette méthode est de ne pas tenir compte du fait que la fonction $\varphi_{2}(\phi)$ doit être régulière. Pour imposer une certaine régularité à l'évolution angulaire de flux $\varphi_{2 i}$, on rajoute à la somme précédente un terme régularisateur qui tend à lisser les fluctuations de flux et donc à linéariser cette évolution comme l'ont présentée Beck et al. [7] : 


$$
\begin{aligned}
S=\sum_{i=0}^{N}( & \left.y_{i}-\theta_{0 i}\right)^{2}+ \\
& +\gamma \sum_{i=1}^{N-2}\left(\varphi_{2 i+2}-2 \varphi_{2 i+1}+\varphi_{2 i}\right)^{2} .
\end{aligned}
$$

Nous avons choisi ici un terme régularisateur du second ordre qui minimise la dérivée seconde de la fonction $\varphi_{2}(\phi)$ car, comme nous le verrons plus loin - paragraphe 4 - l'absence de ce terme conduit à des oscillations de cette fonction si l'on effectue une inversion à partir d'un profil bruité de température.

L'influence sur l'inversion du choix du paramètre régularisateur $\gamma$ introduit en (22) sera étudiée plus loin, ce qui permettra d'adopter non pas une valeur empirique mais une valeur optimale.

Les flux sont alors solution de l'équation :

$$
\frac{\partial S}{\partial \varphi_{2 l}}=0 \text {. }
$$

Ce qui peut encore s'écrire sous la forme :

$$
G\left[\varphi_{2}\right]=[p]
$$

avec :

$$
\begin{aligned}
G & =\tilde{B} B+\gamma \tilde{K} K \\
{[p] } & =\tilde{B}[y]
\end{aligned}
$$

$K$ est une matrice tridiagonale de dimension $(N, N)$ :

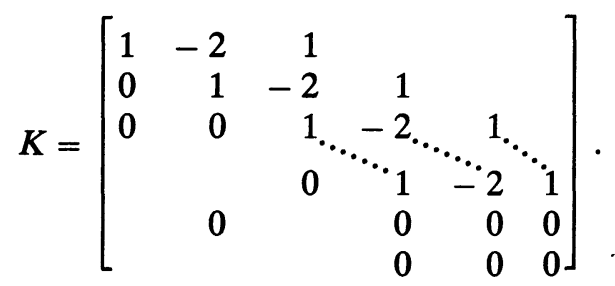

Le système (23) peut être résolu en $\varphi_{2}$ et l'on peut ensuite calculer les coefficients d'échange $h_{i}$ comme précédemment.

3.3 INFLUENCE DE L'INCERTITUDE SUR LE RAYON DE MESURE. - Lorsque l'on dispose de $(N+1)$ mesures $y_{i}$ de la température, éventuellement bruitées, il faut les affecter à un rayon $r_{0}$ d'implantation de la sonde afin de pouvoir calculer les coefficients $h_{i}$ par la méthode ci-dessus. Nous allons examiner la sensibilité du coefficient moyen de transfert $h_{\mathrm{m}}$ à une incertitude sur $r_{0}$ avec :

$$
\begin{aligned}
\theta_{\mathrm{m}}(r) & =\frac{1}{\pi} \int_{0}^{\pi} \theta(r, \phi) \mathrm{d} \phi \\
\theta_{0 \mathrm{~m}} & =\theta_{\mathrm{m}}\left(r_{0}\right) \\
\theta_{2 \mathrm{~m}} & =\theta_{\mathrm{m}}\left(r_{2}\right) \\
h_{\mathrm{m}} & =\varphi_{2 \mathrm{~m}} /\left(\theta_{\mathrm{e}}-\theta_{2 \mathrm{~m}}\right) \\
\varphi_{2 \mathrm{~m}} & =\frac{1}{\pi} \int_{0}^{\pi} \varphi_{2}(\phi) \mathrm{d} \phi .
\end{aligned}
$$

Par le changement de fonction (7) et par intégration de l'équation de la chaleur (1) et de ses conditions limites (4) et (6) sur l'intervalle $[0,2 \Pi]$, on obtient le système différentiel suivant :

$$
\left\{\begin{array}{l}
\frac{\partial^{2} \theta_{\mathrm{m}}}{\partial r^{2}}+\frac{1}{r} \frac{\partial \theta_{\mathrm{m}}}{\partial r}=0 \\
\theta_{\mathrm{m}}=0 \text { en } r=r_{1} \\
\lambda \frac{\partial \theta_{\mathrm{m}}}{\partial r}=\varphi_{2 \mathrm{~m}} \text { en } r=r_{2}
\end{array}\right.
$$

dont la solution est :

$$
\theta_{\mathrm{m}}(r)=\frac{r_{2}}{\lambda} \ln \left(\frac{r}{r_{1}}\right) \varphi_{2 \mathrm{~m}} .
$$

En écrivant la relation (25) en $r_{0}$ et en $r_{2}$, le coefficient moyen $h_{\mathrm{m}}$ peut être calculé à l'aide de sa définition (24) :

$$
h_{\mathrm{m}}=\frac{\lambda}{r_{2}\left(\frac{\theta_{\mathrm{e}}}{\theta_{0 \mathrm{~m}}} \ln \frac{r_{0}}{r_{1}}-\ln \frac{r_{2}}{r_{1}}\right)} .
$$

En dérivant cette relation par rapport à $r_{0}$, en gardant $\theta_{0 \mathrm{~m}}$ constant (résultat des mesures), on obtient la relation suivante :

$$
\frac{1}{h_{\mathrm{m}}} \frac{\partial h_{\mathrm{m}}}{\partial r_{0}}=-\frac{1}{r_{0}\left(\ln \frac{r_{0}}{r_{1}}-\frac{\theta_{0 \mathrm{~m}}}{\theta_{\mathrm{e}}} \ln \frac{r_{2}}{r_{1}}\right)}
$$

$\theta_{0 \mathrm{~m}}$ peut alors être exprimée en fonction du coefficient moyen $h_{\mathrm{m}}$, de l'écart extrême de température $\theta_{\mathrm{e}}$ et des divers rayons grâce à (26), ce qui nous permet de calculer le coefficient $X_{\mathrm{r}}$ de sensibilité relative de $h_{\mathrm{m}}$ à une incertitude relative sur $r_{0}$ :

$$
X_{\mathrm{r}}=\frac{\Delta h_{\mathrm{m}}}{h_{\mathrm{m}}} / \frac{\Delta r_{0}}{r_{0}}=\frac{1+\mathrm{Bi} \ln 1 / k}{\ln x_{0} / k}
$$

avec :

$$
\mathrm{Bi}=\frac{h_{\mathrm{m}} r_{2}}{\lambda}
$$

Les variations de ce coefficient $X_{\mathrm{r}}$ avec le rayon réduit $x_{0}$, le rapport des rayons interne et externe $k$ et le nombre de Biot $\mathrm{Bi}$ sont représentés sur la figure 4. Les cas de figure les plus intéressants pour une bonne mesure se rapportent aux faibles valeurs de $\mathrm{Bi}$ et $k$ et à une implantation de la sonde de température à proximité de la surface externe du tube.

3.4 INFLUENCE DE L'INCERTITUDE SUR LA TEMPÉRATURE. - Les températures qui sont mesurées sont nécessairement bruitées. Nous allons examiner l'incidence sur le coefficient moyen $h_{\mathrm{m}}$ d'une incertitude sur la température moyenne $\theta_{0 \mathrm{~m}}$. 


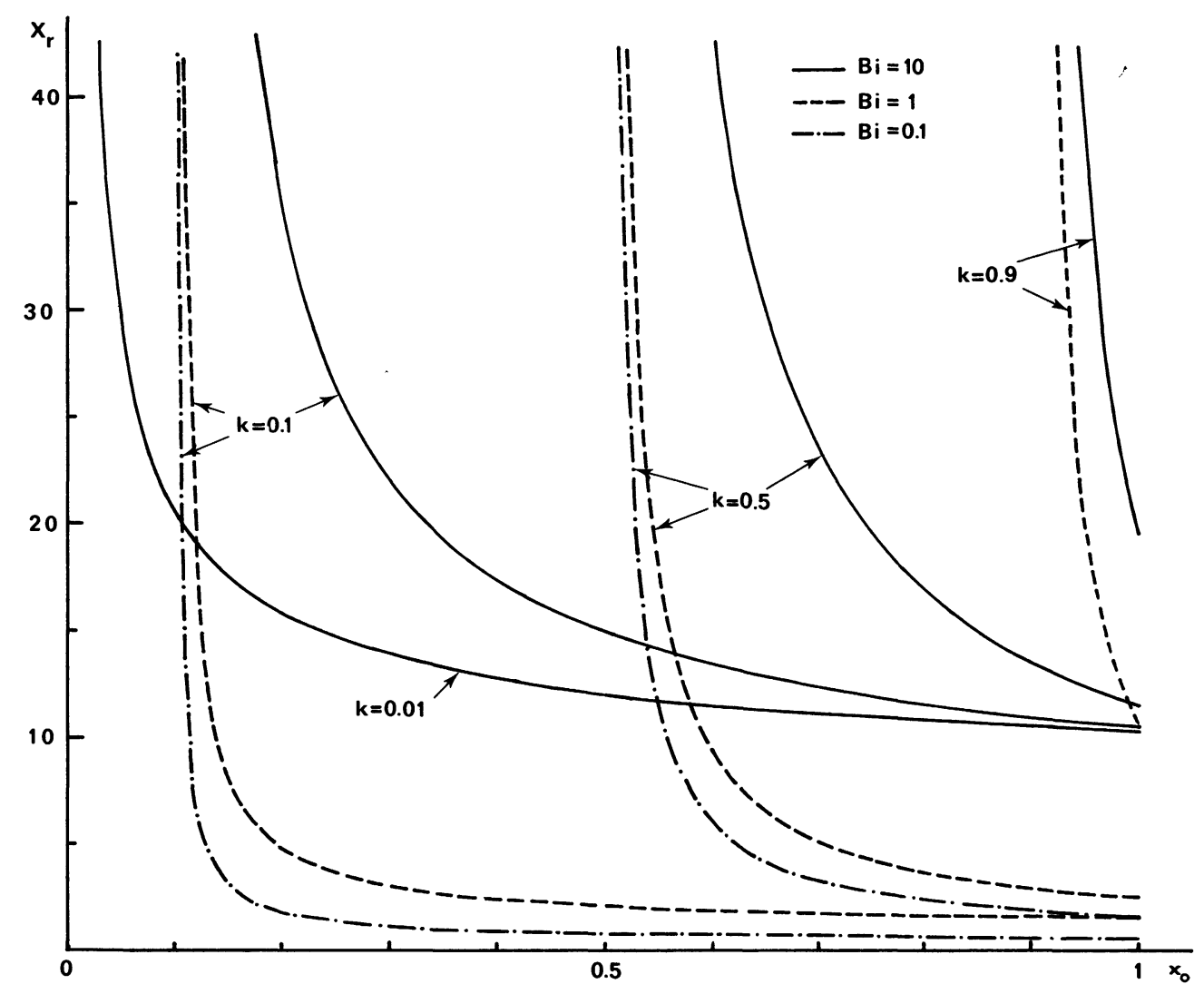

Fig. 4. - Sensibilité à la localisation de la mesure de température.

[Sensitivity to temperature measurement position.]

De la même façon que précédemment il est possible de calculer le coefficient de sensibilité relative de $h_{\mathrm{m}}$ à une incertitude relative sur les températures :

$$
X_{T}=\frac{\Delta h_{\mathrm{m}}}{h_{\mathrm{m}}} / \frac{\Delta \theta_{0 \mathrm{~m}}}{\theta_{\mathrm{e}}}=\frac{(1+\mathrm{Bi} \ln 1 / k)^{2}}{\mathrm{Bi} \ln x_{0} / k} .
$$

Les variations de ce coefficient $X_{T}$ avec le rayon réduit $x_{0}$, le facteur de forme $k$ du cylindre et le nombre de Biot sont données sur la figure 5. Pour chaque valeur de $k$ il existe un nombre de Biot $\mathrm{Bi}_{k}$ pour lequel $X_{T}$ est minimum.

Remarquons que dans le meilleur des cas - faible valeur de $k$, Bi proche de $\mathrm{Bi}_{k}$ et sonde de température implantée près de la surface - une erreur relative de $1 \%$ (normée par l'écart extrême $\theta_{\mathrm{e}}$ ) donne une erreur de $4 \%$ sur l'échange moyen $h_{\mathrm{m}}$.

\section{Résultats du modèle inverse sur des données simulées.}

4.1 Méthodologie. - Beck et al. [7] ont défini trois cas tests qui permettent de voir comment se comporte le modèle avec ou sans bruit. Il s'agit des cas où la fonction à identifier en surface présente une variation en impulsion de Dirac, puis en échelon et enfin en fonction triangle. Dans notre problème, où la fonction à identifier est $h(\phi)$, ces trois cas ont été simulés (Figs. 6, 7, 8a et b) pour 18 pas angulaires de $10^{\circ}$, différents niveaux de bruit et différentes valeurs du paramètre régularisateur $\gamma$.

Le modèle inverse a également été étudié pour le cas de convection forcée présenté précédemment (Fig. 9).

Pour chacun de ces cas la démarche adoptée a été la suivante :

- $h(\phi)$ et ses valeurs discrétisées $h_{i}$ sont données et l'on calcule la répartition exacte des températures $\theta_{0}(\phi)$ au rayon $r_{0}$ choisi par le modèle direct ;

- la température exacte $\theta_{0 i}$ est bruitée avec un bruit aléatoire $\varepsilon_{i}$ d'écart-type $\sigma$ ce qui produit une simulation $y_{i}$ des mesures expérimentales ;

- le modèle inverse décrit précédemment est alors mis en cuvre en introduisant un paramètre régularisateur $\gamma$, ce qui nous donne une estimation $\hat{h}_{i}$ de la valeur du coefficient de transfert en surface.

4.2 Discussion. - Remarquons tout d'abord que pour les quatre cas étudiés, il n'y a aucune différence, lorsque le bruit $\sigma$ est nul, entre les valeurs directes $\varphi_{2 i}$ et $h_{i}$ et les valeurs estimées après inversion $\hat{\varphi}_{2 i}$ et $\hat{h}_{i}$ lorsque l'on ne régularise pas 


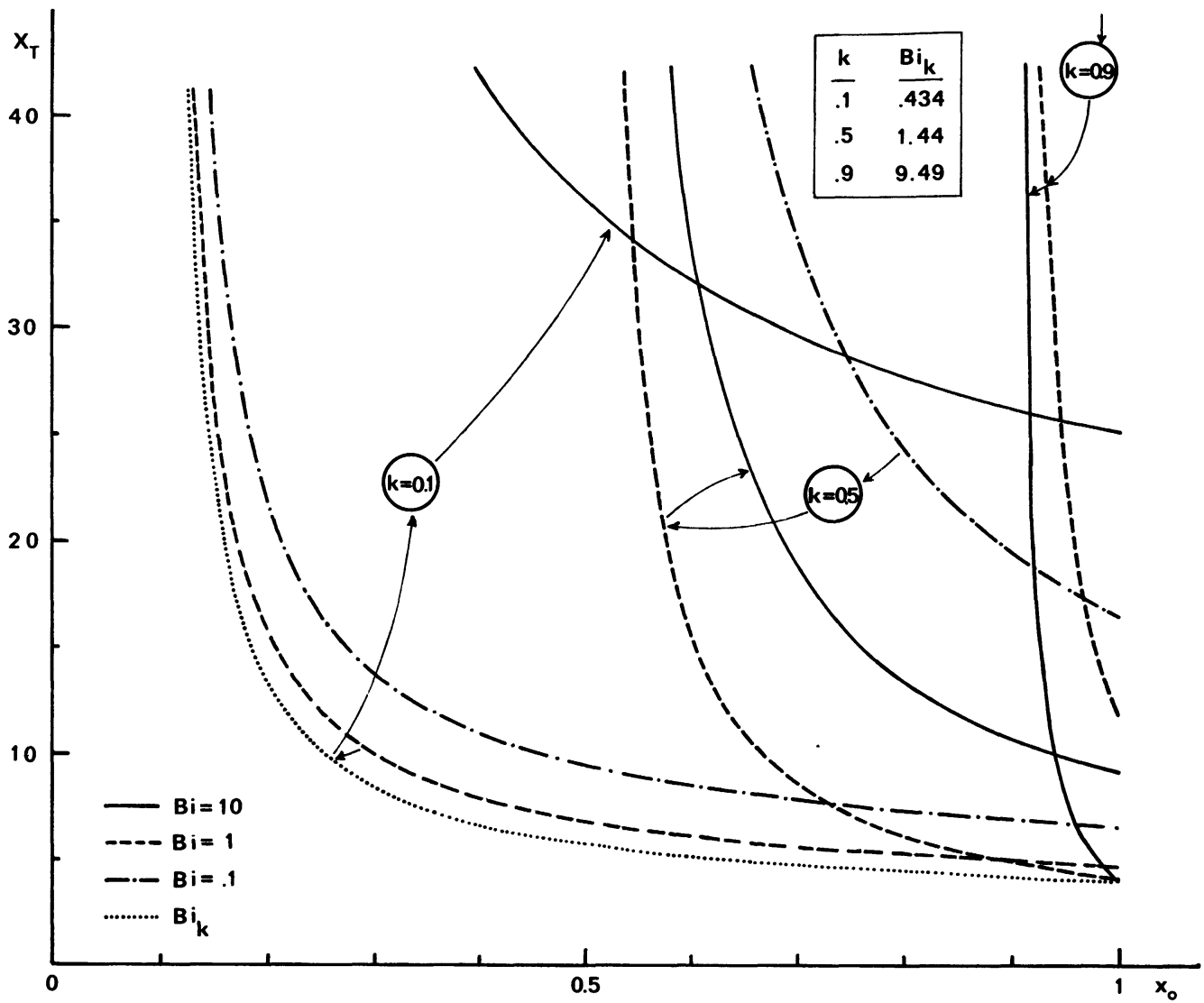

Fig. 5. - Sensibilité à l'incertitude sur la température.

[Sensitivity to temperature error.]

$(\gamma=0)$ ou lorsque l'inversion directe présentée en 3.1 est utilisée.

Cela résulte de la linéarité de la relation température-densité de flux donnée par le modèle direct relation (14) ou (21). L'erreur déterministe au sens de Beck est donc nulle lorsque le bruit est nul, ce qui est un avantage du modèle analytique.

Dès que l'on passe à un bruit de $0,05^{\circ} \mathrm{C}$ d'écarttype, les deux premiers cas qui présentent une variation «dure » du coefficient $h$ (Figs. 6 et 7) ne peuvent plus être correctement approximés par le modèle inverse (que ce soit en $h$ ou en $\varphi_{2}$ ) :

- Si $\gamma$ est nul ou faible $\left(10^{-8}\right)$ les valeurs estimées se mettent en osciller dès le changement brutal de la valeur de $h_{i}$ à $80^{\circ}$ et ce, de façon très importante dans le cas de l'échelon.

- Si $\gamma$ est plus fort $\left(10^{-4}\right)$, les oscillations sont réduites mais les variations brusques sont extrêmement amorties et réparties sur une large plage angulaire.

- Si $\gamma$ est grand $(=1)$, c'est le terme régularisateur qui domine dans la somme (22) dont la minimisation produit une évolution quasi linéaire de $\varphi_{2}$ (Fig. 7b) et donc hyperbolique de $h$ (Fig. 7a).

Ces estimations n'ont alors plus grand chose à voir avec les valeurs directes.
Dans les deux derniers cas qui présentent une variation plus «douce » de $h$ (Figs. 8 et 9), un bruit identique produit les mêmes oscillations en l'absence de régularisation. Par contre, pour des valeurs intermédiaires de $\gamma\left(10^{-8}\right.$ et $\left.10^{-4}\right)$, l'accord entre valeurs directes et valeurs estimées devient très satisfaisant et ce particulièrement dans le dernier cas (Fig. 9).

Notons également que dans ce cas, même un bruit dix fois plus fort $-\sigma$ de $0,5^{\circ} \mathrm{C}-$ fournit encore l'allure générale du profil direct de $h$.

Notre modèle inverse semble donc adapté à des mesures expérimentales de $h$ dans les cas réels où son évolution est toujours « douce » et régulière.

4.3 CHOIX DU COEFFICIENT RÉGULARISATEUR. Le paramètre régularisateur $\gamma$ doit être optimisé si l'on désire traiter des mesures expérimentales. Nous avons vu plus haut que sa valeur optimum dépendait du niveau de bruit. La meilleure valeur de $\gamma$ est celle qui minimise le résidu moyen $R_{h}$ des coefficients de transfert :

$$
R_{h}^{2}=\frac{1}{N} \sum_{i=1}^{N}\left(\hat{h}_{i}-h_{i}\right)^{2}
$$




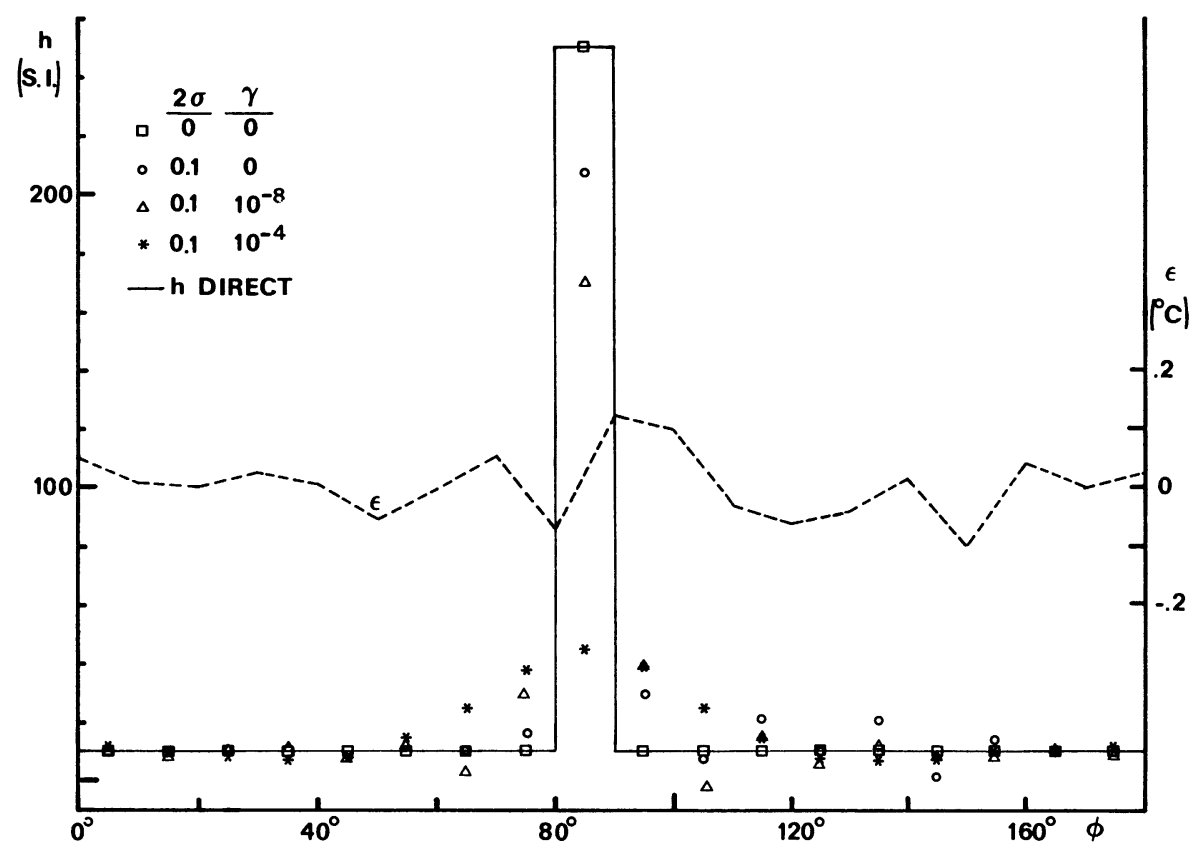

a)

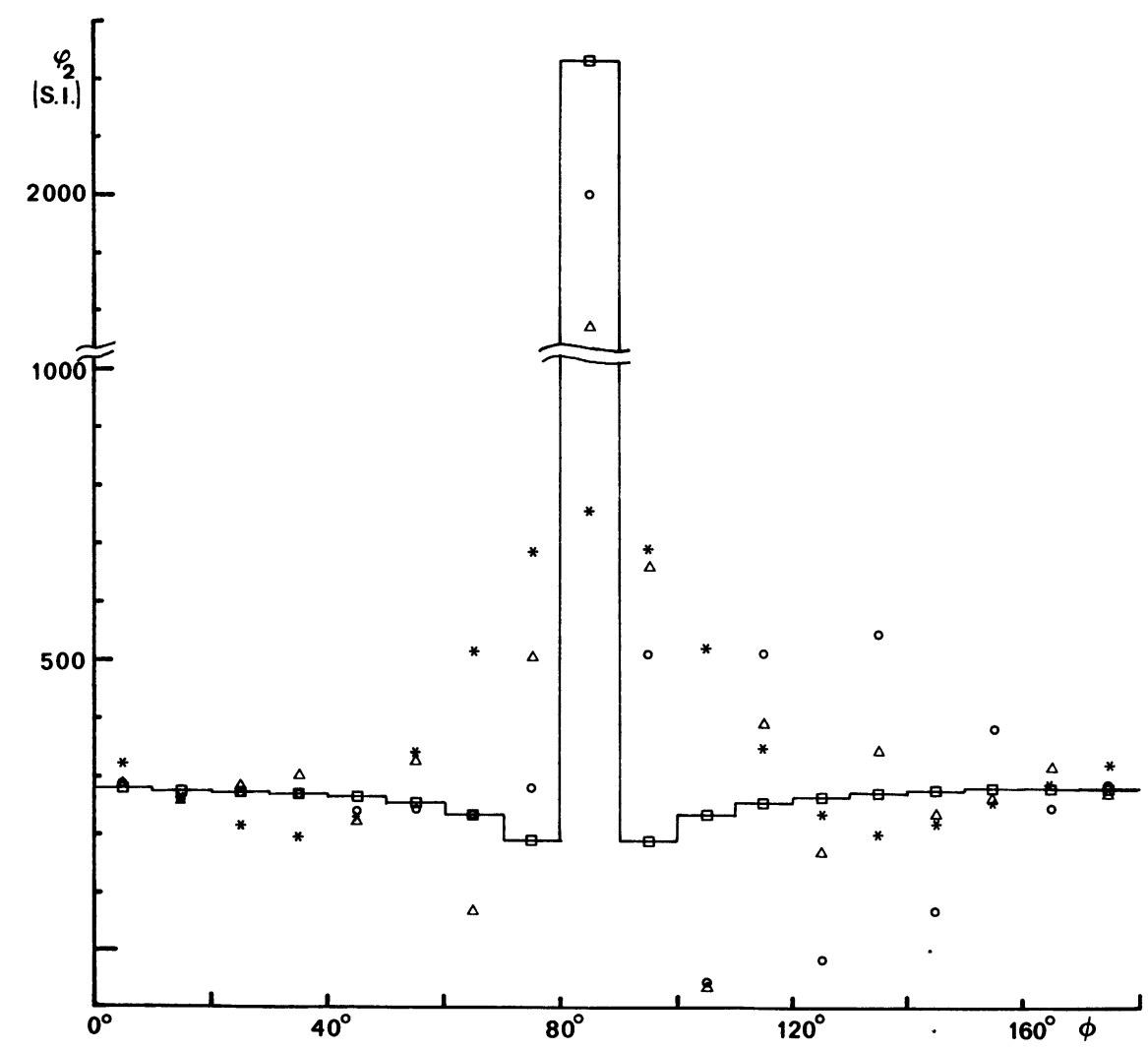

b)

Fig. 6. - $(\mathrm{a}, \mathrm{b})$ Problème inverse, réponse à une impulsion de Dirac de coefficient de transfert. [Inverse problem, response to a Dirac-shaped pulse of heat-transfer coefficient.] 
Il est également possible, à partir des densités de flux $\hat{\varphi}_{2 i}$ fournies par le modèle inverse de recalculer, par le modèle direct, des températures estimées $\hat{\theta}_{0 i}$ aux points de mesure et d'évaluer un résidu moyen des températures :

$$
R_{T}^{2}=\frac{1}{N} \sum_{i=0}^{N}\left(\hat{\theta}_{0 i}-Y_{i}\right)^{2}
$$

Nous avons calculé le résidu $R_{h}$ en fonction de $\gamma$ pour un bruit $\sigma$ de $0,05^{\circ} \mathrm{C}$ dans le dernier cas convection forcée sur le cylindre - La valeur optimum de $\gamma$ est :

$$
\gamma=1,5 \times 10^{-6}
$$

avec :

$$
\begin{aligned}
& R_{h}=2,68 \mathrm{~W} \mathrm{~m}^{-2} \mathrm{~K}^{-1} \\
& R_{T}=0,047 \mathrm{~K}
\end{aligned}
$$

Notons que le résidu de température, c'est-à-dire la part de la température non expliquée par le modèle, est du même ordre de grandeur que l'écarttype du bruit de la mesure lorsque $\gamma$ est optimum, ce qui est satisfaisant.

\section{Résultats expérimentaux.}

5.1 Montage EXPÉRIMENTAL. - Un cylindre de résine époxy (conductivité de $0,26 \mathrm{~W} \mathrm{~m}^{-1} \mathrm{~K}^{-1}$ ) de $500 \mathrm{~mm}$ de longueur, de $8 \mathrm{~mm}$ de rayon intérieur et $16 \mathrm{~mm}$ de rayon extérieur a été obtenu par moulage puis tournage sur un tube de cuivre de diamètres 14/16. Trois thermocouples en chromel-alumel de $0,12 \mathrm{~mm}$ de diamètre ont été implantés parallèlement aux génératrices du cylindre, les soudures, décalées les unes par rapport aux autres d'un angle d'environ $120^{\circ}$, étant localisées dans le plan de

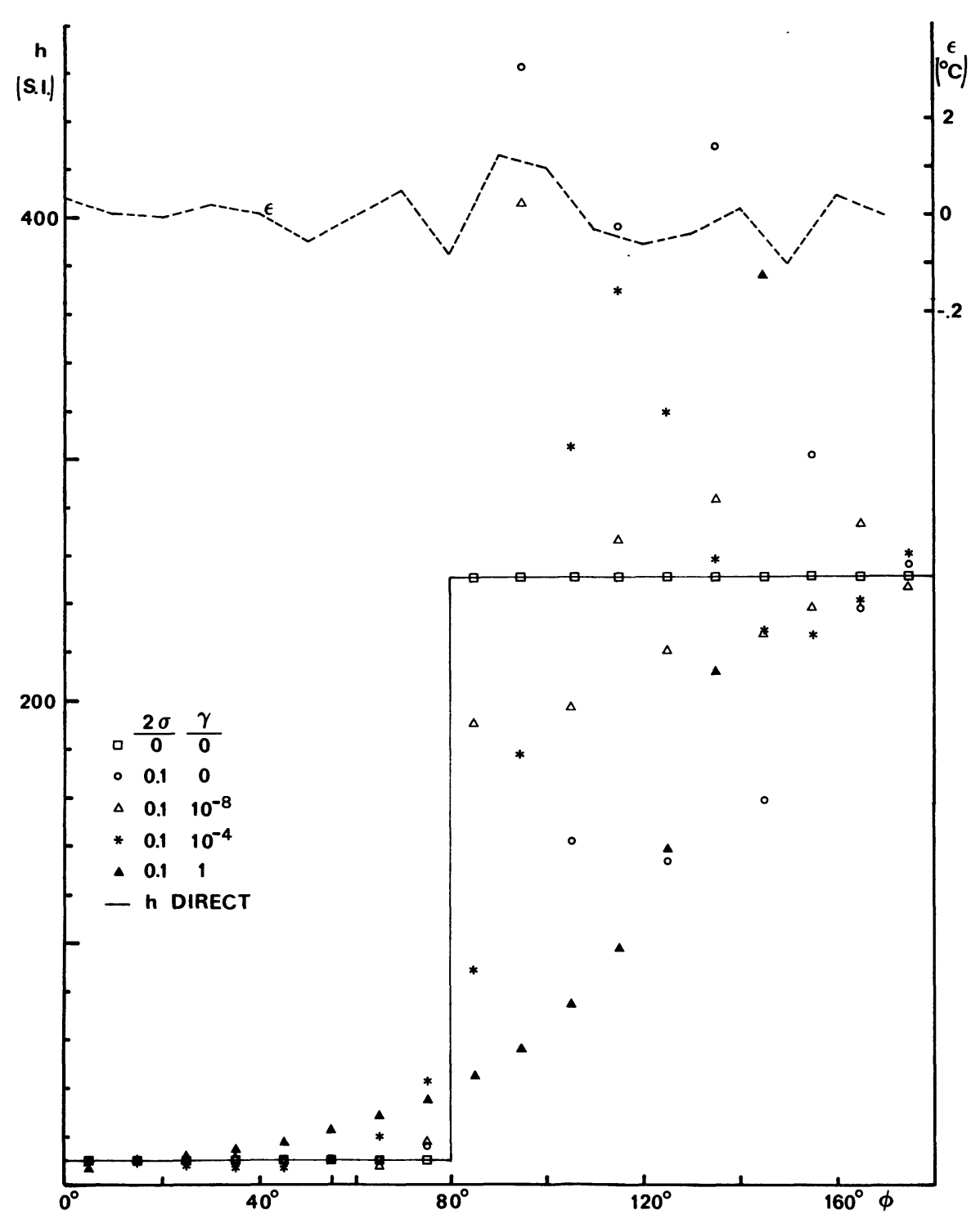

a) 


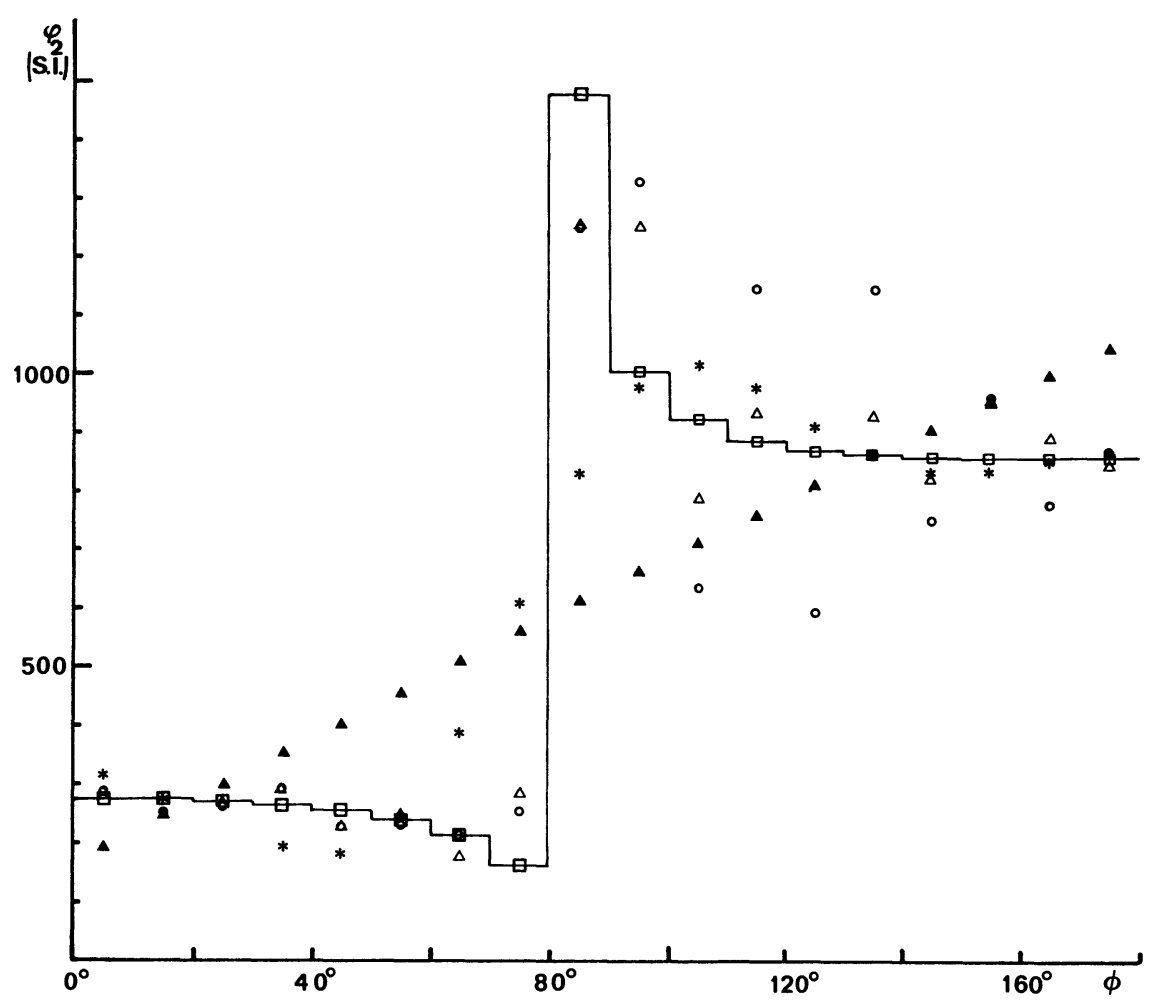

b)

Fig. 7. - $(\mathrm{a}, \mathrm{b})$ Problème inverse, réponse à un échelon de coefficient de transfert.

[Inverse problem, response to a step-function of heat-transfer coefficient.]

symétrie transverse du cylindre. Leurs rayons nominaux d'implantation étaient les suivants :

$$
\begin{aligned}
& r_{0}=14 \mathrm{~mm} \\
& r_{0}^{\prime}=12 \mathrm{~mm} \\
& r_{0}^{\prime \prime}=10 \mathrm{~mm} .
\end{aligned}
$$

Un débit d'eau chaude à $65^{\circ} \mathrm{C}$, produit par un thermostat à circulation, à travers le tube central en cuivre, assurait la condition limite interne. La température du tube de cuivre était également mesurée par un thermocouple implanté dans la section transverse.

Le cylindre de mesure a été placé dans la veine d'une soufflerie de section $400 \times 400 \mathrm{~mm}$ dont le taux de turbulence était de l'ordre de $1 \%$. La température de l'air en amont du cylindre était également mesurée par un thermocouple.

5.2 RÉSUltats Des MESURES. - Les mesures ont été effectuées pour une vitesse d'air de $30,7 \mathrm{~m} / \mathrm{s}$, ce qui correspond à un nombre de Reynolds de 64250 . Une rotation du cylindre d'un pas de $10^{\circ}$ permettait d'obtenir un nouveau point de mesure pour les trois sondes une fois le régime permanent établi, ce qui prenait environ 30 à $40 \mathrm{~min}$.

A cause de la durée de l'expérience et de la chaleur dégagée par le moteur de la soufflerie, la température ambiante ne restait pas constante entre chaque mesure. Il a donc fallu ramener toutes les mesures à un même écart des températures extrêmes $\theta_{\mathrm{e}}$. Nous avons opté pour une correction unidirectionnelle de la température :

$$
y_{i}^{*}=y_{i}+\frac{\mathrm{Bi} \ln x_{0} / k}{1+\mathrm{Bi} \ln 1 / k}\left(\theta_{\mathrm{e}}-\theta_{\mathrm{ei}}\right)
$$

$\theta_{\mathrm{e}}$ est la moyenne des écarts extrêmes de température mesurés $\theta_{\mathrm{e} i}$ pour chaque mesure $y_{i}$ de $\theta_{0 i}$ et $y_{i}^{*}$ est la valeur corrigée de $\theta_{0 i}$, le nombre de Biot $\mathrm{Bi}$ étant calculé sur un transfert moyen $h_{\mathrm{m}}$ de $175 \mathrm{~W} \mathrm{~m}^{-2} \mathrm{~K}^{-1}$.

Les profils bruts et corrigés de température pour chaque sonde sont présentés (Figs. 10a, b et c). On remarque l'effet de lissage dû à la correction $1 \mathrm{D}$.

5.3 LoCAlisation des MESURES. - Il est nécessaire d'affecter un rayon à chaque profil $y_{i}{ }^{*}$ afin de pouvoir mettre en ouvre le modèle inverse explicité plus haut. Pour ce faire, nous avons détruit par tournage le cylindre de mesure et avons mesuré les rayons minima et maxima de chaque soudure $0,8 \mathrm{~mm}$ de diamètre environ - sur une photographie de la coupe prise à l'aide d'un objectif macroscopique. Cela nous a permis de donner pour chaque rayon un intervalle d'incertitude.

La relation (25), portant sur les valeurs moyennes 


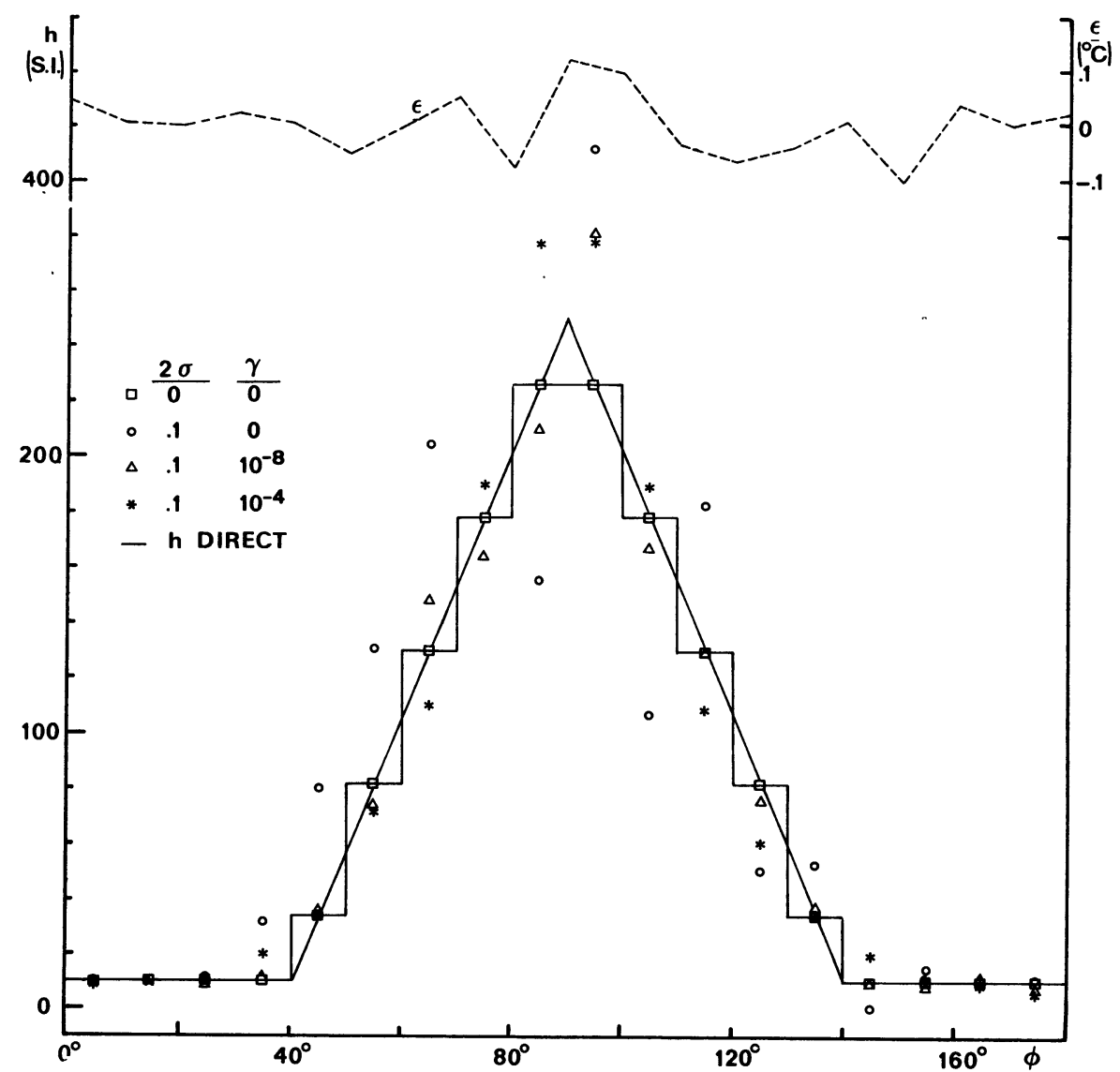

a)

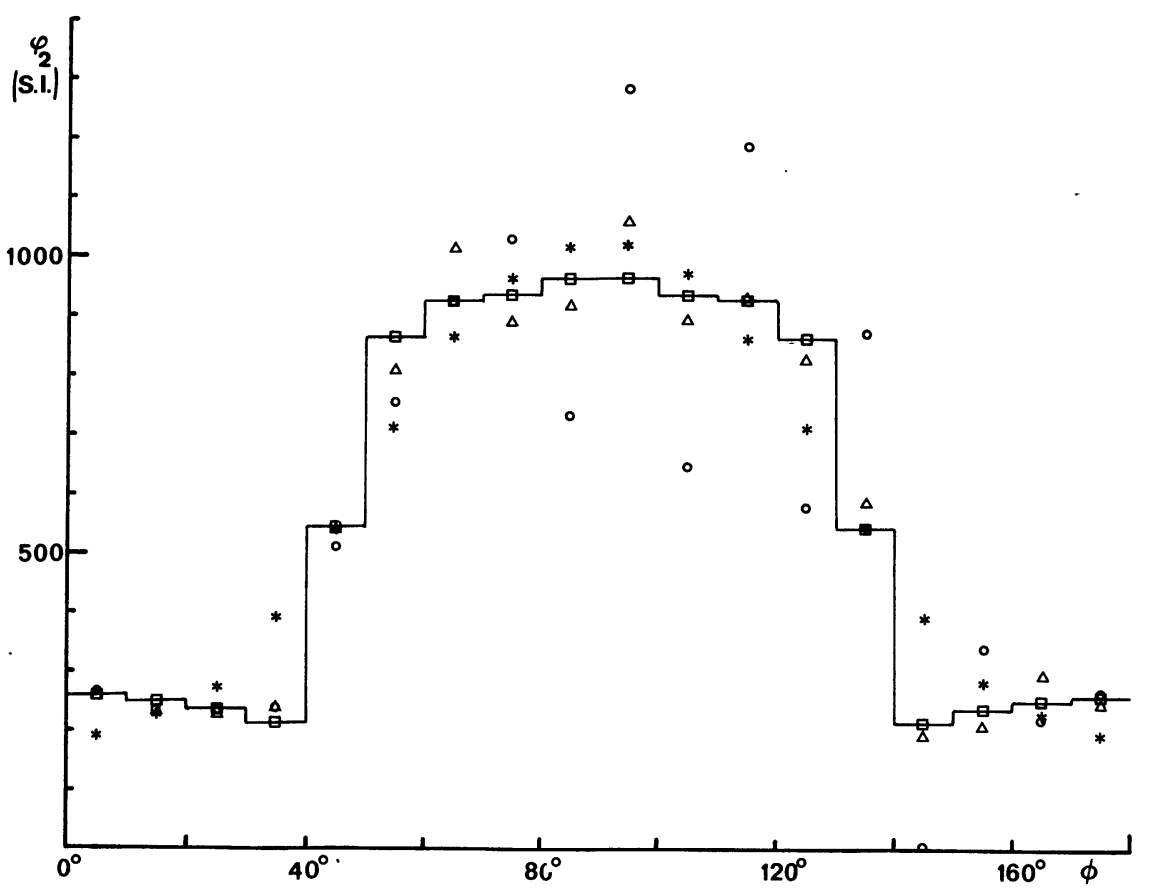

b)

Fig. 8. - $(\mathrm{a}, \mathrm{b})$ Problème inverse, réponse à une fonction triangle de coefficient de transfert. [Inverse problem, response to a triangle-function of heat-transfer coefficient.] 


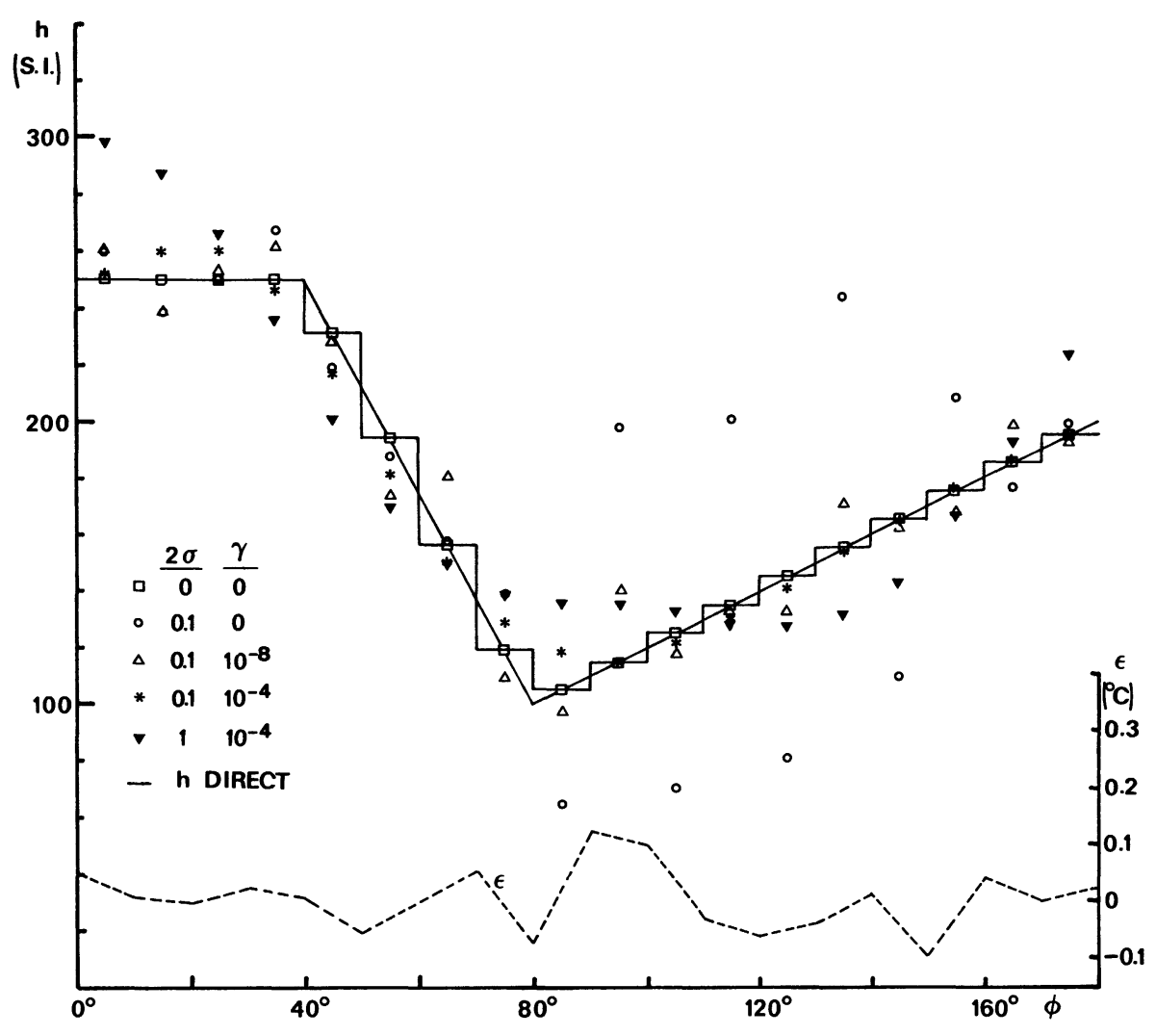

Fig. 9. - Problème inverse, réponse à une évolution de coefficient de transfert typique de la convection forcée.

[Inverse problem, response to a typical forced convection evolution of the heat-transfer coefficient.]

de la température et du flux, écrite pour deux rayons différents $r_{0}$ et $r_{0}^{\prime}$, rend possible la diminution des plages d'incertitudes. En faisant le rapport des deux relations, on obtient :

$$
\frac{\theta_{0 \mathrm{~m}}^{\prime}}{\theta_{0 \mathrm{~m}}}=\frac{\ln r_{0}^{\prime} / r_{1}}{\ln r_{0} / r_{1}}=\frac{q_{0}^{\prime}}{q_{0}}
$$

Cette relation définit également les rayons homologues $r_{0}$ et $r_{0}^{\prime}$, des sondes externe et centrale, qui fournissent la même densité moyenne de flux $\varphi_{2 \mathrm{~m}}$.

Le rapport des températures moyennes exactes $\theta_{0 \mathrm{~m}}$ est égal au rapport des moyennes des valeurs mesurées et corrigées $y_{i}^{*}$ si le bruit $\varepsilon_{i}$ est un aléa centré. Ce rapport peut donc être calculé par intégration numérique. Le rapport des logarithmes des rayons réduits de deux sondes de température est alors connu ; cela permet de ramener les incertitudes sur $q_{0}^{\prime}$ et $q_{0}^{\prime \prime}$ à des incertitudes sur $q_{0}$ et donc de diminuer l'incertitude sur le rayon $r_{0}$. En pratique, cette dernière est divisée par deux.

Nous avons utilisé le modèle inverse pour calculer les profils $h(\phi)$ présentés sur les figures $11 \mathrm{a}$, b et c pour les valeurs minimum, centrale et maximum de $r_{0}$ ainsi que pour les valeurs homologues des rayons $r_{0}^{\prime}$ et $r_{0}^{\prime \prime}$.
5.4 Discussion. - Les profils de coefficient de transfert obtenus grâce à la méthode inverse sont extrêmement sensibles à l'incertitude sur le rayon d'implantation des sondes de température. On retrouve ici les résultats du modèle unidimensionnel présenté en 3.3 : nos mesures ont été effectuées pour un nombre de Biot voisin de 10 et un coefficient de forme $k$ de 0,5 qui nous permettent d'évaluer les coefficients de sensibilité au rayon $X_{\mathrm{r}}$ de 14,20 et 35 pour les trois sondes en partant de la sonde la plus externe. Cela signifie par exemple qu'une erreur de $0,25 \mathrm{~mm}$ sur le rayon d'implantation de cette dernière sonde produit une incertitude de l'ordre de $25 \%$ sur le coefficient moyen de transfert $h_{\mathrm{m}}$. L'unique façon d'éliminer cette incertitude sur la position du thermocouple serait de mesurer le flux total quittant le cylindre par une méthode calorimétrique et d'utiliser ensuite la relation (25) pour évaluer son rayon.

Malgré les valeurs élevées du coefficient de sensibilité à l'incertitude sur la température $X_{T}$ - ici de 11,15 et 28 (voir Fig. 5) - l'incertitude sur la température, qui est voisine de $0,05^{\circ} \mathrm{C}$ pour un écart extrême $\theta_{\mathrm{e}}$ de $40^{\circ} \mathrm{C}$, n'entraîne qu'une incertitude de $1,4 \%$ sur le transfert moyen $h_{\mathrm{m}}$ lorsque l'on calcule ce dernier à l'aide des températures mesurées par le thermocouple extérieur. 


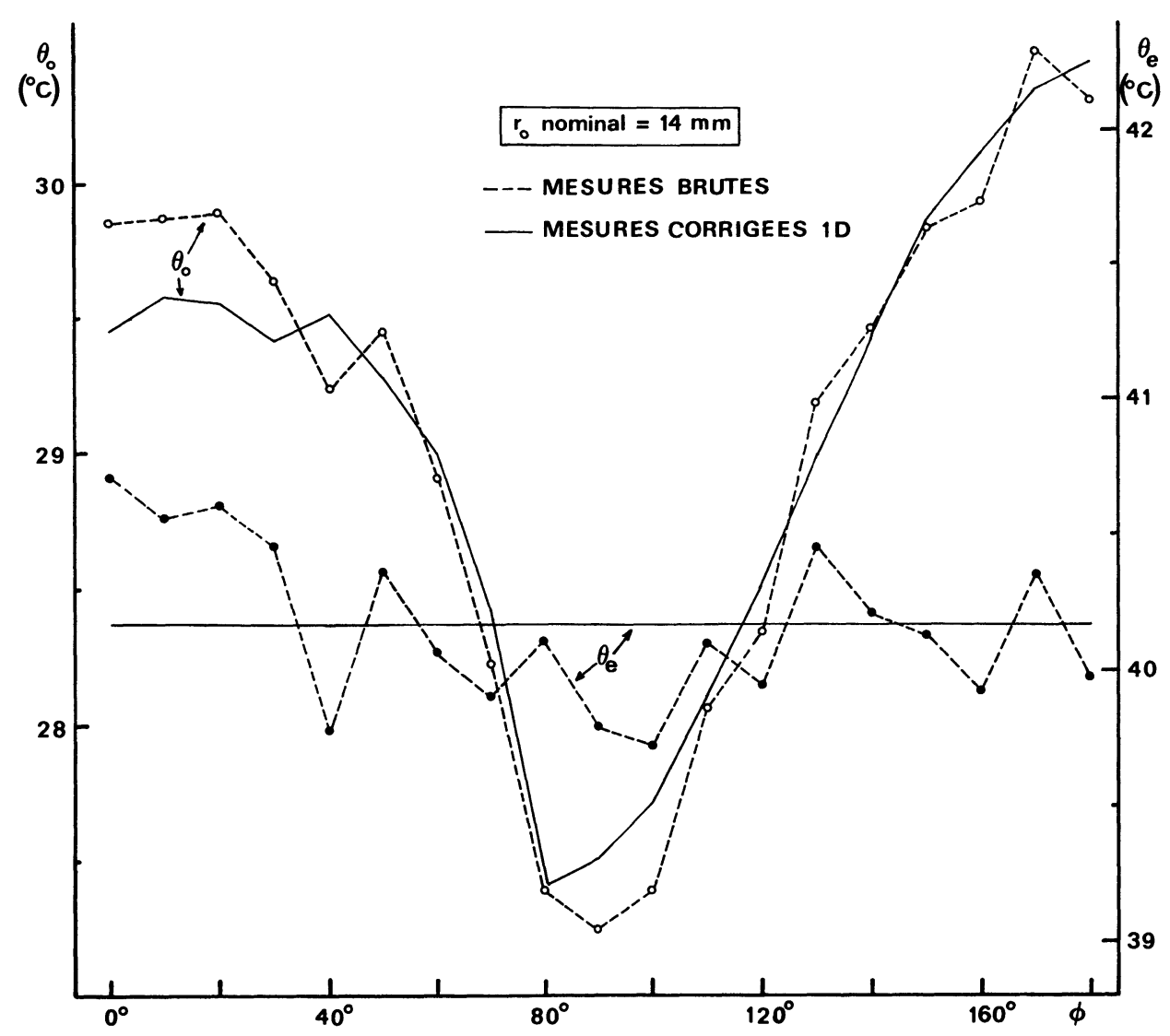

a)

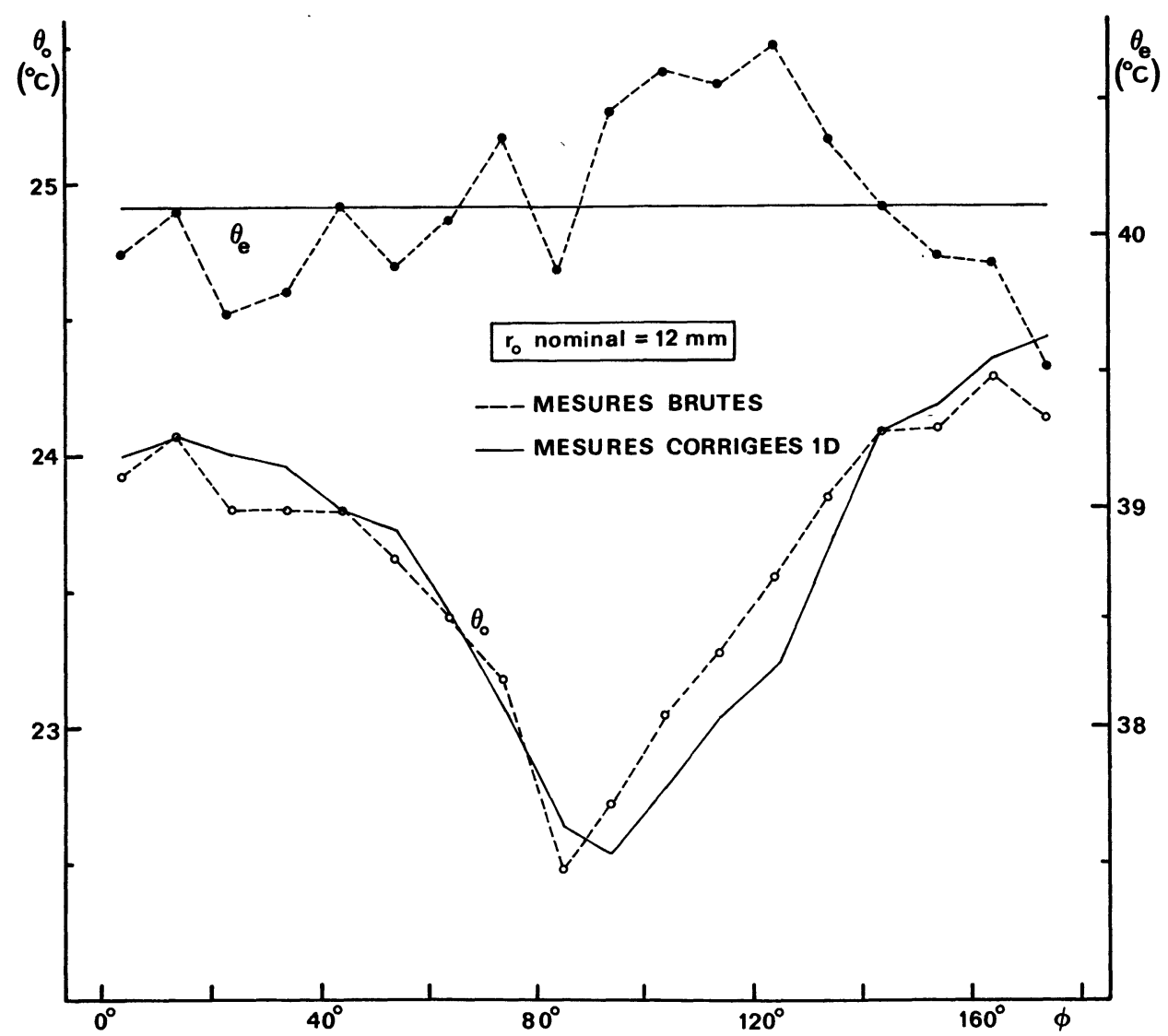

b) 


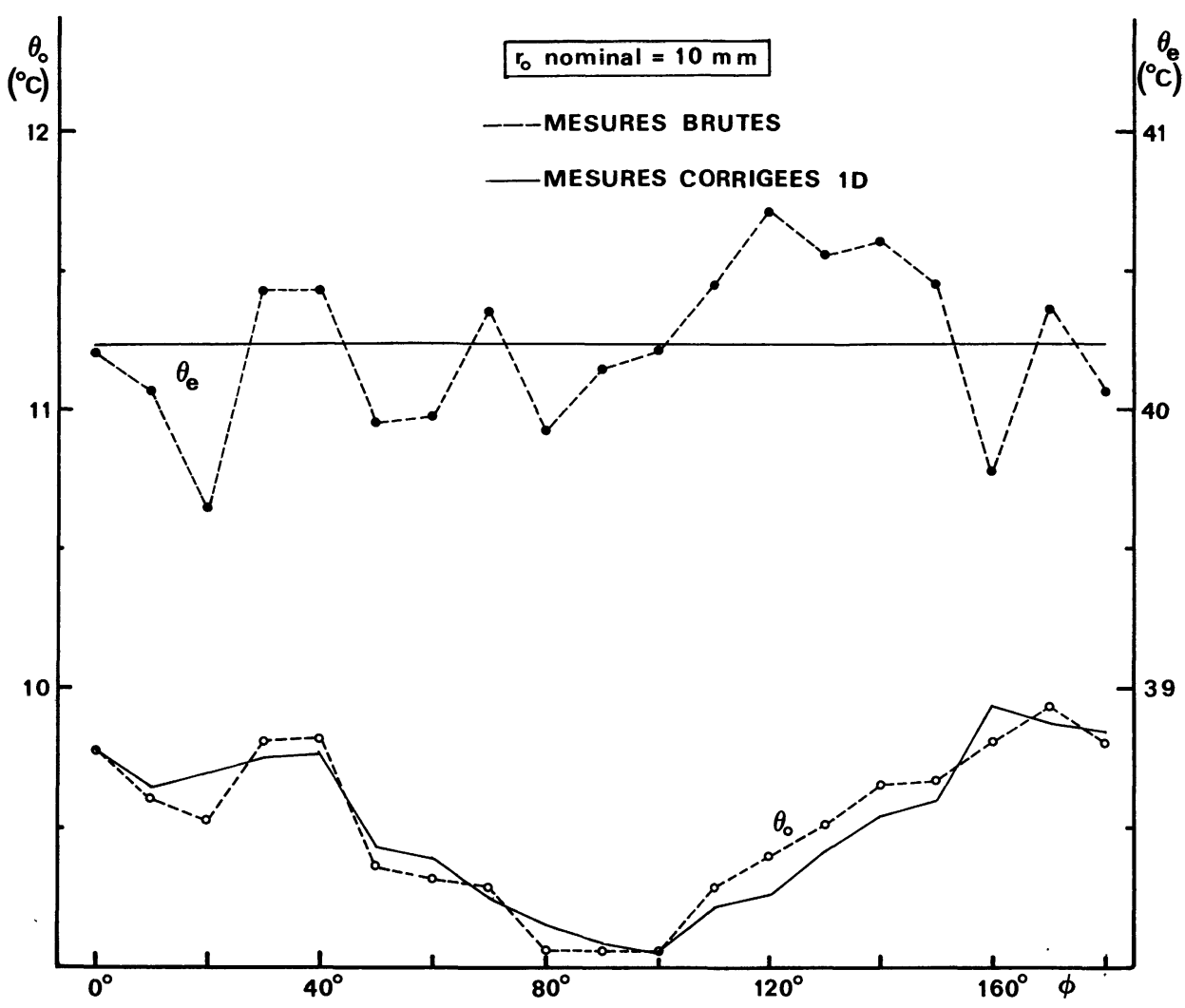

c)

Fig. 10. - $(\mathrm{a}, \mathrm{b}, \mathrm{c})$ Températures expérimentales.

[Experimental temperatures.]

Les profils du coefficient $h$ représentés sur les figures 11 mettent en évidence la bonne concordance des résultats obtenus à partir des profils indépendants de température relevés aux rayons homologues central $r_{0}^{\prime}$ et externe $r_{0}$. Le coefficient $h$ présente bien une valeur constante sur les 50 premiers degrés, puis un minimum voisin de $90^{\circ}$ suivi d'une remontée quasi linéaire jusqu'à $180^{\circ}$.

Les résultats obtenus avec la sonde interne $r_{0}^{\prime \prime}$ s'écartent notablement des autres profils entre 0 et $50^{\circ}$. Cela provient certainement, d'une part d'une plus forte sensibilité locale au bruit lorsque l'on se rapproche du rayon interne $r_{1}$ et d'autre part du comportement de la résine utilisée dans la gamme de température mesurée par cette sonde $\left(54\right.$ à $\left.57^{\circ} \mathrm{C}\right)$. Une mesure en continu de la capacité thermique de la résine a mis en évidence une transformation vitreuse endothermique centrée sur $62^{\circ} \mathrm{C}$, c'est-àdire à une température inférieure à celle du tube de cuivre central $\left(65\right.$ à $\left.66^{\circ} \mathrm{C}\right)$. Cette transformation n'est plus mise en œuvre lorsque le régime permanent est atteint ; par contre, toute fluctuation de température induit une fluctuation du degré d'avancement de la transformation qui crée ainsi un terme local - source ou puits de chaleur - dans l'équation (1) et perturbe le champ des températures au voisinage du rayon $r_{0}^{\prime \prime}$ de la sonde interne.

REVUE DE PHYSIQUE APPLIQUÉE. - T. 24, N` 7, JUILLET 1989
Les profils du coefficient $h$ représentés sur les figures 11 ont été calculés à l'aide d'une conductivité thermique $\lambda$ de $0,26 \mathrm{~W} \mathrm{~m}^{-1} \mathrm{~K}^{-1}$ pour la résine. Les mesures de capacité thermique mentionnées plus haut et de diffusivité thermique nous ont permis d'accéder à la valeur de la conductivité qui est pratiquement constante et égale à $0,20 \mathrm{~W} \mathrm{~m}^{-1} \mathrm{~K}^{-1}$ entre 30 et $53^{\circ} \mathrm{C}$. La moyenne des profils obtenus avec cette nouvelle valeur de $\lambda$, pour les rayons homologues de 14,015 et $12,62 \mathrm{~mm}$ est donnée à la figure 12.

Sur la figure $11 \mathrm{~b}$, on observe que la pente $h(\phi)$ n'est pas nulle au point d'arrêt aval, ce qui est incompatible avec l'hypothèse de symétrie par rapport à la direction du vecteur vitesse amont. Cela peut d'abord provenir du fait que les valeurs corrigées $y_{i}^{*}$ de la température continuent à croître lorsqu'on se rapproche de ce point traduisant ainsi une dissymétrie de l'écoulement causée par exemple par un effet dynamique de convection naturelle dans la zone de sillage du cylindre horizontal. D'autre part, cette pente non nulle peut être due à l'absence de mesure des points symétriques. Pour les prendre en compte nous avons ajouté à la somme $S(22)$ un terme régularisateur $S^{\prime}$ du premier ordre sur les deux intervalles extrêmes où justement la dérivée seconde discrétisée n'avait pu être évaluée : 


$$
S^{\prime}=\left(\varphi_{22}-\varphi_{21}\right)^{2}+\left(\varphi_{2 N}-\varphi_{2 N-1}\right)^{2}
$$

Sans changer la valeur précédente de $\gamma$, les résultats de l'inversion (Fig. 12) montrent un infléchissement notable du profil au voisinage de $180^{\circ}$.

Nous avons reporté, sur la même figure, l'évolution angulaire du coefficient de transfert obtenue par Giedt [8] pour un nombre de Reynolds Re voisin du nôtre et celle mesurée par Achenbach [9] pour un nombre de Reynolds un peu supérieur. Les mesures de ces deux auteurs, portant sur le nombre de Nusselt, ont été transcrites en $h$ à l'aide du rayon $r_{2}$ et de la conductivité thermique de l'air. Les trois profils présentent la même évolution qualitative. On retrouve l'évolution quasi linéaire sur l'aval du cylindre chez Achenbach avec un transfert au point d'arrêt aval nettement supérieur à celui du point amont. Pour les faibles taux de turbulence

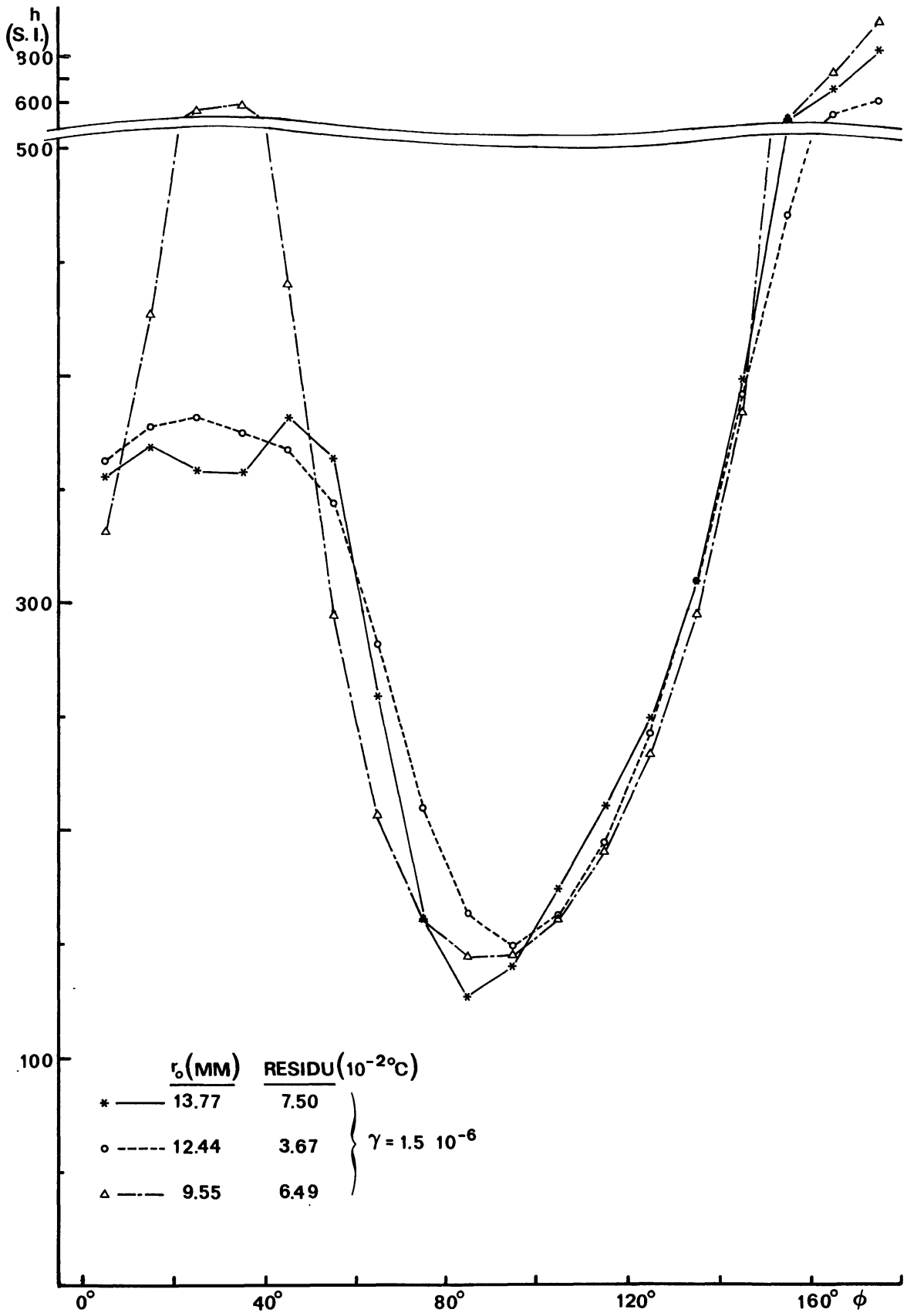

a)

Fig. 11. - Coefficients de transfert mesurés, conductivité $\lambda=0,26 \mathrm{~W} \mathrm{~m}^{-1} \mathrm{~K}^{-1}$, a) rayons homologues minima ; b) rayons homologues moyens ; c) rayons homologues maxima.

[Measured heat-transfer coefficients, conductivity $\lambda=0.26 \mathrm{~W} \mathrm{~m}^{-1} \mathrm{~K}^{-1}$, a) homologous lowest radii ; b) homologous medium radii ; c) homologous highest radii.] 


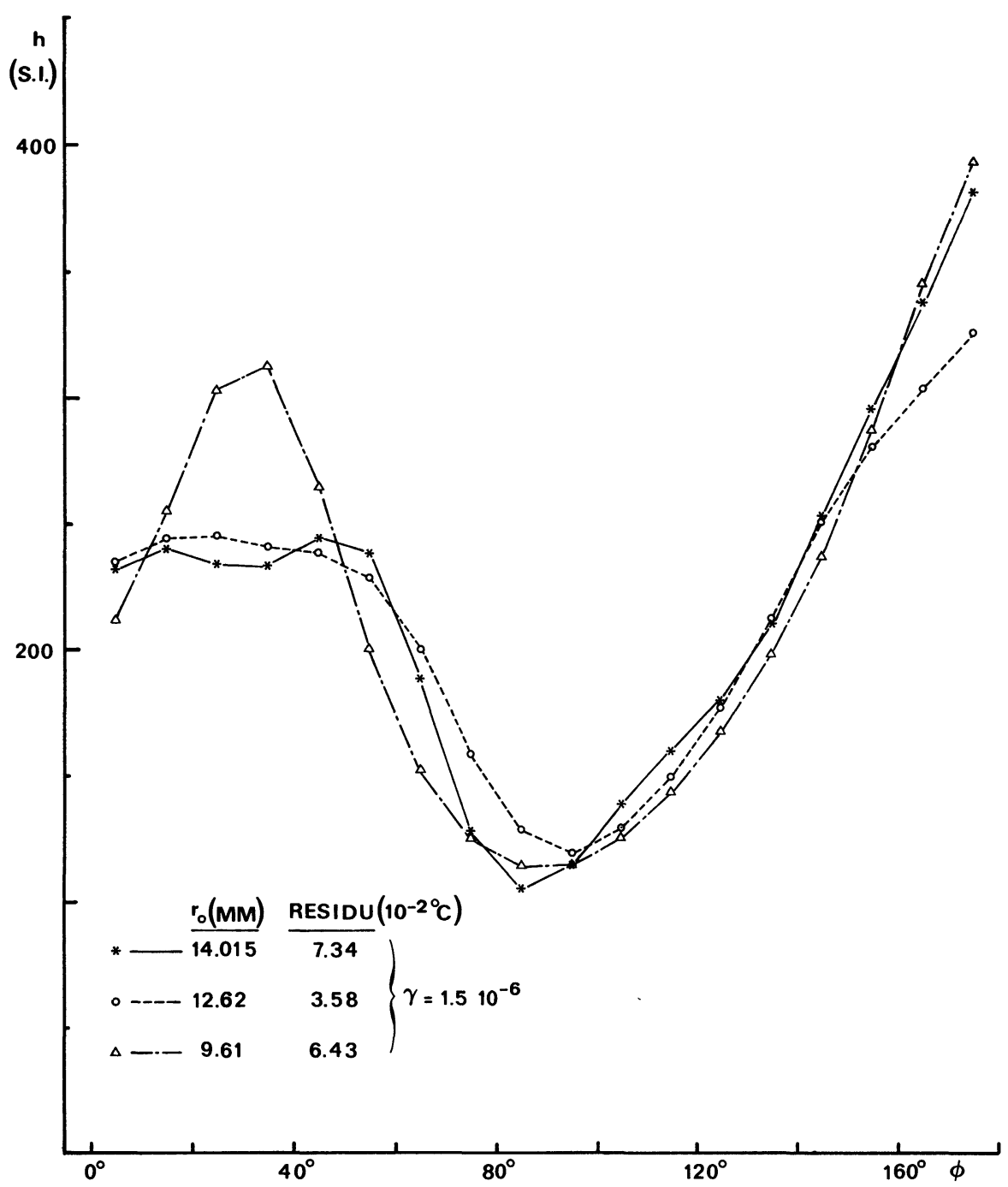

b)

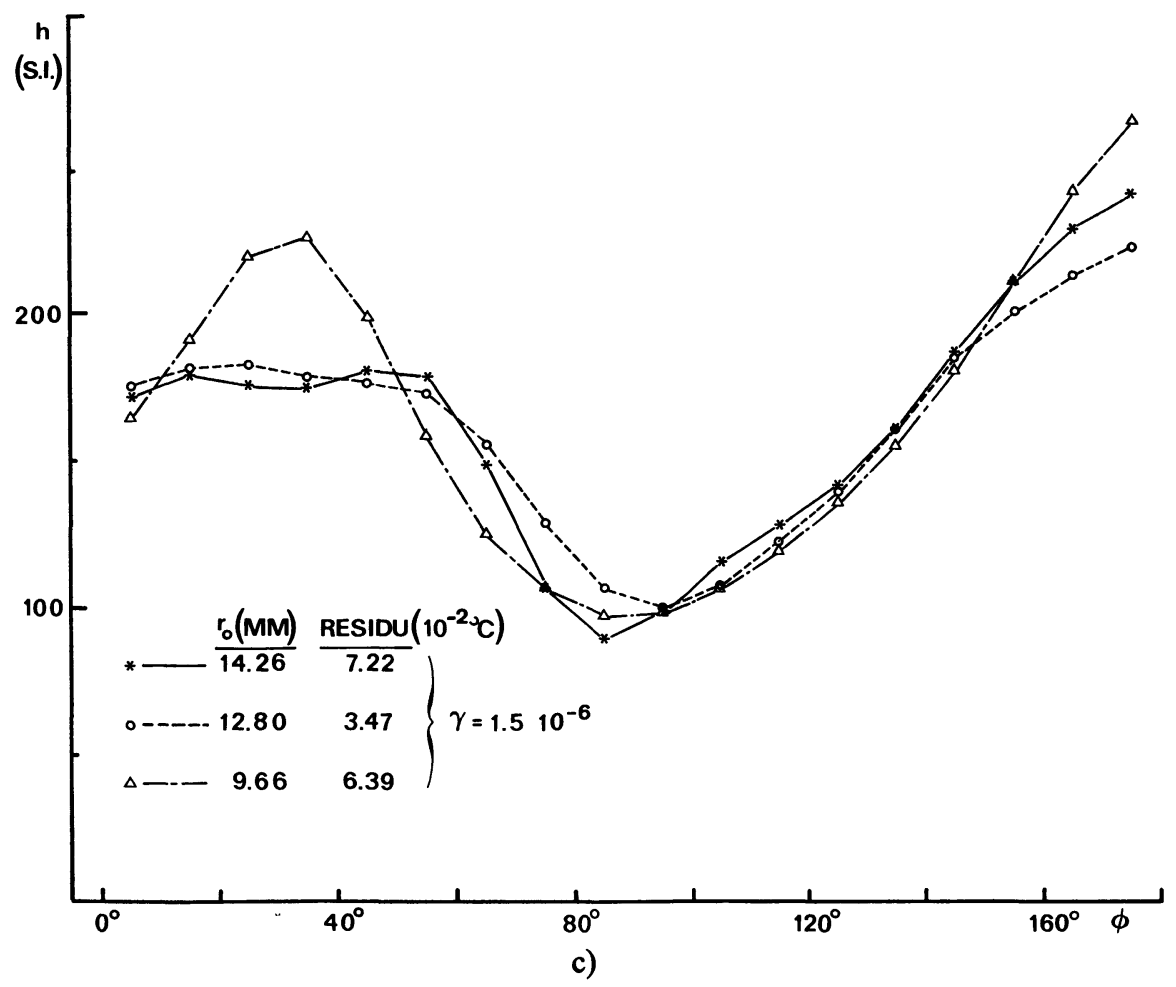




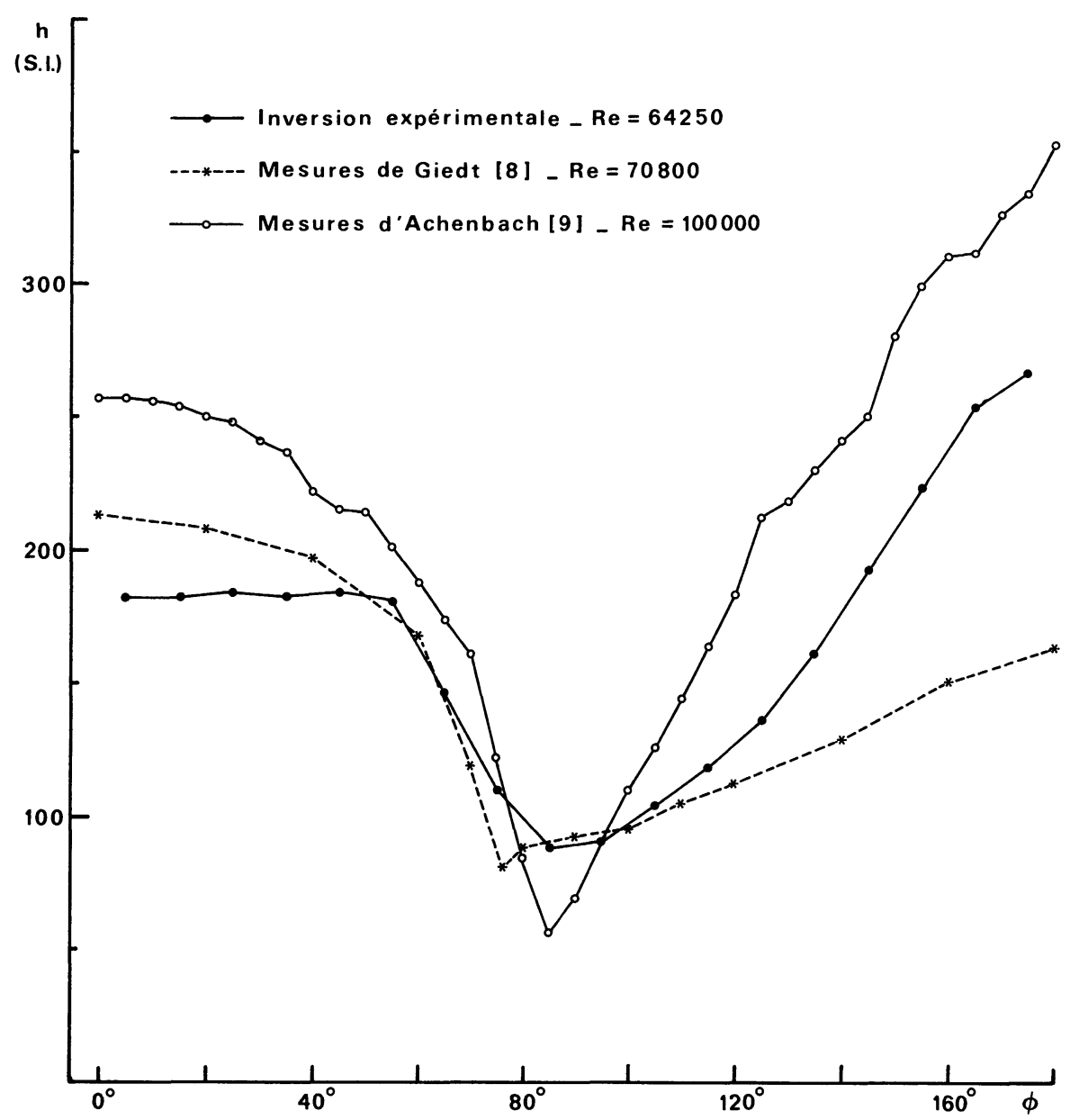

Fig. 12. - Comparaison avec des mesures de la littérature.

[Comparison with previous experimental results.]

Zukauskas [1] propose des corrélations locales pour le nombre de Nusselt qui varient en $\operatorname{Re}^{0,5}$ au point d'arrêt amont, en $\operatorname{Re}^{0,83}$ au point d'arrêt aval et en $\operatorname{Re}^{0,6}$ sur le transfert moyen en régime subcritique correspondant à nos mesures. Il est donc pratiquement impossible d'isoler l'influence du nombre de Reynolds pour faciliter la comparaison entre les trois courbes de la figure 12. Les différences peuvent provenir des conditions expérimentales - taux de turbulence, facteur de blocage, condition limite surfacique [1] - mais aussi des limitations inhérentes aux méthodes de mesure basées sur des bilans comme nous l'avons expliqué en introduction.

Notons, pour illustrer ce dernier point que, à la différence de nos mesures, les profils [8] et [9] présentent un minimum très marqué au voisinage du point de décollement de la couche limite $\left(\simeq 80^{\circ}\right)$. Cela vient du fait que les mesures de $h$ sont faites par un bilan local qui rend difficile la prise en compte de la conduction tangentielle à la paroi. En effet ces méthodes modifient le champ de température au cœur du cylindre et ceci de façon différente pour chaque point de mesure : chaque point de mesure ne correspond donc pas au même problème. Inversement, notre technique expérimentale permet d'éliminer ce problème.

\section{Avantages et inconvénients de la méthode.}

Les avantages de cette méthode inverse analytique pour l'obtention de l'évolution angulaire du coefficient de transfert sur un cylindre, par rapport à une méthode basée sur des bilans, sont les suivants :

- pas de perturbation des lignes de flux : l'homogénéité du cylindre de mesure est conservée ;

- partie expérimentale aisée (pas de réglage de résistances de compensation);

- estimation des pertes inutile.

Par rapport à une méthode inverse non analytique, les avantages sont les suivants :

- maîtrise de la sensibilité aux incertitudes de température et de positionnement, a priori pour les moyennes angulaires et a postériori pour les valeurs locales ;

- pas d'incertitude sur le rayon de la mesure si le 
flux total échangé est mesuré par une méthode calorimétrique ;

- inversion exacte sur des données non bruitées ;

- temps de calcul très faible pour l'inversion -

$5 \mathrm{mn}$ sur un D.P.S.6 de Bull - à cause de la linéarité du modèle direct : la minimisation de la fonctionnelle ne réclame pas la mise en œuvre d'un processus itératif ;

- obtention aisée de la valeur optimisée du paramètre régularisateur.

D'autre part, l'extension à un cas où le profil de coefficient de transfert n'est pas symétrique ne pose pas de problème.

Le seul inconvénient par rapport à une méthode numérique est la nécessité d'utiliser un matériau solide stable dont la conductivité thermique reste constante sur une large plage de température.

\section{Conclusion.}

Nous avons établi un modèle analytique permanent bidimensionnel de transfert de chaleur permettant de calculer la température interne à partir d'un profil surfacique du coefficient de transfert sur un cylindre. L'influence du pas angulaire de discrétisation, ainsi que la faisabilité de la méthode inverse ont été étudiées sur un cas test. Un modèle inverse, prenant en compte un terme régularisateur, pour résoudre le problème du bruit de la mesure, a été présenté. La sensibilité de la méthode aux erreurs sur le rayon de mesure et sur la température a été étudiée pour les moyennes angulaires du coefficient de transfert. Des simulations ont été effectuées en choisissant des profils bruités comme entrées du modèle inverse, ce qui a permis de l'essayer sur quatre cas tests. La valeur du paramètre régularisateur a été optimisée sur une de ces simulations.

Des mesures expérimentales sur trois rayons ont été obtenues. Elles ont confirmé l'importance de la connaissance précise du rayon de mesure et ont été cohérentes entre elles. Un profil expérimental issu de cette méthode inverse a été comparé à un profil obtenu par deux autres auteurs dans des conditions voisines. Les avantages et inconvénients de la méthode ont été examinés.

\section{Bibliographie}

[1] ZuKausKas A., ZiUgzda J., Heat transfer of a cylinder in crossflow, Ed. G. F. Hewitt (Hemisphere Pub. Corp., Springer Verlag, New York) 1985.

[2] Martin M. G., Gosse J., Etude de la convection thermique autour des cylindres par un écoulement pulsé, C. R. Acad. Sc. Paris Série A 266 (1968) 1107.

[3] Degiovanni A., Sinicki G., Gery A., Laurent M., Un modèle de résistance thermique de contact en régime permanent, Rev. Gén. Therm. 267 (1984) 161-175.

[4] Keltner N. R., BecK J. V., Unsteady surface element method, J. Heat Transfer 103 (1981) 759-764.

[5] OzIsIK M. N., Heat conduction (John Wiley and Sons, New York) 1980.
[6] Courant R., Hilbert D., Methods of mathematical physics (John Wiley and Sons, New York) 1, Chap. 3 (1953).

[7] BeCK J. V., BlaCKWell B., St-Clair C. J. Jr., Inverse heat conduction - Ill-posed problems (John Wiley and Sons, New York) 1985.

[8] GIEDT W. H., Investigation of variation of point unitheat-transfer coefficient around a cylinder normal to an air stream, Trans. ASME 71 (1949), cité par KREITH F., Principles of heat-transfer (Intext Educational Pub., New York) 1973.

[9] ACHENBACH E., Total and local heat transfer from a smooth circular cylinder in cross-flow at high Reynolds number, Int. J. Heat Mass Transfer 18 (1975) $1387-1396$. 